\title{
Three-dimensional stability theory of a continuous beam with axisymmetric Kapchinskij-Vladimirskij distribution
}

\author{
Tai-Sen F. Wang \\ Los Alamos National Laboratory, Los Alamos, New Mexico 87545, USA
}

(Received 8 November 2003; published 17 February 2004)

\begin{abstract}
This work is a three-dimensional stability study based on the modal analysis for a continuous beam with an axisymmetric Kapchinskij-Vladimirskij (KV) distribution. The analysis is carried out selfconsistently within the context of linearized Vlasov-Maxwell equations and electrostatic approximation. The emphasis is on investigating the coupling between longitudinal and transverse perturbations in the high-intensity region. The interaction between the transverse modes supported by the KV distribution and the "usual transverse modes" is examined. We found two classes of "coupling modes" that would not exist if longitudinal and transverse perturbations are treated separately. We also found that some transverse modes can interact among themselves through longitudinal perturbation to cause instability. The effects of wall impedance on beam stability is also studied and numerical examples are presented.
\end{abstract}

DOI: $10.1103 /$ PhysRevSTAB.7.024201

PACS numbers: 29.27.Bd, 41.75.-i, 52.35.-g, 52.59.Sa

\section{INTRODUCTION}

There is increasing interest in the applications of charged-particle beams such as heavy-ion fusion, spallation neutron sources, tritium production, and transmutation of waste radioactive materials, calling for increased beam intensity [1,2]. Experimental accelerator facilities, like the University of Maryland Electron Ring [3] currently under construction, have been designed for higher and higher beam intensities as well. The rise of the demanded beam current into the space-charge dominated regime necessitates a better understanding of beam stability. For example, in a customary stability analysis of a continuous beam in an accelerator or storage ring, longitudinal and transverse effects are treated separately [4-8], an approximation that is valid because spacecharge forces are relatively weak and characteristic frequencies differ by orders of magnitude. A new situation arises in a proposed heavy-ion fusion facility which has a beam current several orders of magnitude higher than that in a conventional accelerator or storage ring. Spacecharge forces are large and all frequencies are of the order of the plasma frequency, so that the separated treatment of longitudinal and transverse perturbations may not be applicable. Thus, in the very high-intensity regime, an appropriate beam stability theory should be based on a three- or two-dimensional kinetic-theory analysis to account for the possible coupling between longitudinal and transverse perturbations. Some three-dimensional calculations have been carried out before, but the considerations were limited to laminar beams or beams with very small radial motion of particles or modes with only surface perturbation on the beams $[7,9,10]$. These kinds of models appear too simple for a strongly focused beam in a heavy-ion fusion driver in which most particles have appreciable betatron oscillations in the radial direction to support many transverse modes not found in a laminar or nearly laminar beam.

Concern of the longitudinal-transverse coupling effect was raised more than two decades ago in the heavy-ion fusion studies. Since then, some investigations have been exploited in attempt to address the issue by improving the earlier stability theories for laminar beams or nearly laminar beams $[11,12]$. In a study of two-dimensional, axisymmetric perturbations in a beam with a Kapchinskij-Vladimirskij (KV) distribution [13], an instability caused by the coupling between longitudinal and transverse motion was discovered in theory [12]. Later computer simulations confirmed the prediction [14-16] and found this kind of instability to be a mechanism for energy exchange between longitudinal and transverse motions in the beams with high anisotropy in temperature. The instability was then linked to the one investigated by Harris [17] for plasmas with anisotropy in velocity distribution. These findings and many related fine papers published afterward [18-29] mark success in exploring the intense beam stability. However, to date, the rigorous three-dimensional stability analysis based on the kinetic theory is still left in an incomplete stage and further progress remains to be pursued. The motivation of the present work is to revisit the classical problem of three-dimensional beam stability by investigating a simple model and using minimal approximation(s).

Among all models of charged-particle beams, the KV distribution is probably the most used for self-consistent equilibria. Because of its simplicity, it has been used for various computations and stability analyses involving space-charge effects. In spite of being not very realistic and being more unstable than a realistic distribution, it still has many characteristics similar to those of realistic distributions. This can be seen in the modal structure found in computer simulations for more realistic particle 
distributions and in the warm-beam fluid models studied recently [18]. Albeit the KV distribution is very popular, a punctilious, full three-dimensional stability analysis of a $\mathrm{KV}$ beam still has not been carried out, even within the context of electrostatic approximation. This is largely due to the complexity of the mathematical manipulations involved. Nonetheless, the simplicity of the KV distribution function lends itself as yet as the most amiable mathematical model for exact analytical calculation without too many approximations which might neglect or smear the detail of three-dimensional effects. Historically, the first transverse stability study of an axisymmetric KV beam was exploited in Ref. [8] where the radial and the azimuthal transverse modes were investigated under the assumption of no perturbation in the axial direction. Later, the transverse stability of a $\mathrm{KV}$ beam in a periodic focusing channel was analyzed under the same assumption [30]. The investigation in Ref. [30] was followed by several related studies including computer simulations [31-35] and the stability of a breathing axisymmetric KV beam [36,37]. The first analytical stability study including the axial perturbation was carried out in Ref. [12]. However, the discussion there was limited to the azimuthally symmetric modes only. The purpose of this work is to extend the earlier investigation of axisymmetric modes in Ref. [12] to a full three-dimensional kinetic-theory stability study to complete the analysis of the electrostatic stability of a continuous monoenergetic axisymmetric $\mathrm{KV}$ beam. It is hoped that the approach and the results of this work will be helpful in the exploring and understanding of beam stability in nonaxisymmetric geometry.

Although the mathematical calculation involved in the present work is substantially more elaborate, the layout of the present paper and the development of the analysis as well as the mathematical notations will closely follow those in Ref. [12]. In Sec. II, the theoretical model will be delineated and the Vlasov-Poisson equations introduced. The dispersion relation will be derived in Sec. III. Some special cases of the dispersion relation will be discussed in Sec. IV. Numerical examples will be presented in Sec. V. We shall use CGS Gauss units throughout this paper.

\section{THEORETICAL MODEL AND LINEARIZED VLASOV-POISSON EQUATIONS}

We consider an infinitely long nonrelativistic beam of circular cross section with radius $a$ and constant particle density $\rho_{0}$ propagating inside a conducting pipe of radius $b$ and arbitrary wall impedance. A cylindrical coordinate system $(r, \varphi, z)$ is chosen such that the beam is propagating in the positive $z$ direction and the $z$ axis coincides with the central axis of the beam. The equilibrium state of the beam is maintained by a constant linear external transverse focusing force which can be represented as $m_{b} \nu_{o}^{2} r$ where $m_{b}$ is the mass of a beam particle and $\nu_{o}$ is the betatron frequency in the absence of the beam's self-field. Taking the self-field of the beam into account, one finds the relation

$$
\nu^{2}=\nu_{o}^{2}-\left(\omega_{p}^{2} / 2\right)
$$

between the effective betatron frequency of particles $\nu$, and the plasma frequency $\omega_{p}=\left(4 \pi q^{2} \rho_{0} / m_{b}\right)^{1 / 2}$ where $q$ is the charge of a beam particle. We assume the equilibrium distribution of beam particles in phase space is described by the distribution function $f_{0}(\mathbf{x}, \mathbf{v})$ that is a product of the $\mathrm{KV}$ distribution in the transverse direction and a distribution function $f_{z}\left(v_{z}\right)$ for the longitudinal motion, i.e.,

$$
f_{0}(\mathbf{x}, \mathbf{v})=\frac{\rho_{0}}{\pi} \delta\left[v_{\perp}^{2}-\nu^{2}\left(a^{2}-r^{2}\right)\right] f_{z}\left(v_{z}\right),
$$

where $v_{\perp}^{2}=v_{r}^{2}+v_{\varphi}^{2}, v_{r}, v_{\varphi}$, and $v_{z}$ are the particle's radial, azimuthal, and axial speeds, respectively, $\delta(x)$ is the delta function, and $f_{z}\left(v_{z}\right)$ is normalized according to

$$
\int_{-\infty}^{\infty} f_{z}\left(v_{z}\right) d v_{z}=1
$$

Although it is possible to consider a more complex distribution of longitudinal particle velocities, we will concentrate on the discussion of the case of $f_{z}\left(v_{z}\right)=$ $\delta\left(v_{z}-v_{o}\right)$ in the following, where $v_{o}$ is the axial speed of beam particles. The consideration of such a monoenergetic distribution function inevitably neglects the damping effect due to energy spread. Readers have to keep in mind that even a moderate longitudinal energy spread can substantially modify the growth rate as reported in Refs. [27-29].

For the theoretical model considered here, one can easily infer the particle orbit $\left(r^{\prime}, \varphi^{\prime}, z^{\prime}\right)$ in the equilibrium state by solving the equations of motion to find

$$
r^{\prime 2}=r^{2} \cos ^{2}(\nu t)+\left(\frac{v_{\perp}}{\nu}\right)^{2} \sin ^{2}(\nu t)+\frac{r v_{\perp}}{\nu} \sin (2 \nu t) \cos \theta,
$$

$$
\begin{gathered}
\varphi^{\prime}=\varphi+\frac{1}{2}\left\{\sin ^{-1}\left[\frac{h_{\perp}-2 v_{\varphi}^{2}\left(r / r^{\prime}\right)^{2}}{\sqrt{h_{\perp}^{2}-4\left(\nu r v_{\varphi}\right)^{2}}}\right]-\sin ^{-1}\right. \\
\left.\times\left[\frac{h_{\perp}-2 v_{\varphi}^{2}}{\sqrt{h_{\perp}^{2}-4\left(\nu r v_{\varphi}\right)^{2}}}\right]\right\},
\end{gathered}
$$

and

$$
z^{\prime}=v_{z} t+z
$$

where $t$ is the time, $\theta=\cos ^{-1}\left(v_{r} / v_{\perp}\right), h_{\perp}=v_{\perp}^{2}+\nu^{2} r^{2}$, and the initial conditions of $r^{\prime}(t=0)=r, \varphi^{\prime}(t=0)=\varphi$, $z^{\prime}(t=0)=z$, and $\mathbf{v}^{\prime}(t=0)=\mathbf{v}$ were assumed. From the 
equilibrium orbit, one can derive a useful relation

$$
e^{i \varphi^{\prime}}=\left(\frac{e^{i \varphi}}{r^{\prime}}\right)\left(\xi_{1} e^{i \theta}+\xi_{2}\right)
$$

where

$$
\begin{gathered}
\xi_{1}=\left(\frac{v_{\perp}}{\nu}\right) \sin (\nu t), \\
\xi_{2}=r \cos (\nu t),
\end{gathered}
$$

and $i=\sqrt{-1}$.

Our stability study will be carried out within the context of the Vlasov-Maxwell equations and the electrostatic approximation [7,38]. Thus the electric field will follow the description of the Poisson equation and the corresponding magnetic field will be approximately given by $\mathbf{B}=\mathbf{v} \times \mathbf{E} / c$, where $c$ is the speed of light in the vacuum. Further, we limit our discussions of the beam stability to the linear regime. That is we consider small perturbations in the distribution function $f_{1}(\mathbf{x}, \mathbf{v}, t)$ and in the electric potential $\phi_{1}(\mathbf{x}, t)$ described by the linearized Vlasov-Poisson equations

$$
\frac{\partial f_{1}}{\partial t}+\mathbf{v} \cdot \frac{\partial f_{1}}{\partial \mathbf{x}}+\frac{d \mathbf{v}}{d t} \cdot \frac{\partial f_{1}}{\partial \mathbf{v}}=\frac{q}{m_{b}} \nabla \phi_{1} \cdot \frac{\partial f_{0}}{\partial \mathbf{v}},
$$

and

$$
\nabla^{2} \phi_{1}=-4 \pi q \rho_{1}=-4 \pi q \int_{-\infty}^{\infty} \int_{-\infty}^{\infty} \int_{-\infty}^{\infty} f_{1}(\mathbf{x}, \mathbf{v}, t) d^{3} v
$$

where $\rho_{1}$ is the perturbed particle density.

Assuming the perturbed quantities vary in space and time according to

$$
\begin{aligned}
\left\{f_{1}, \rho_{1}, \phi_{1}\right\} & =\{\tilde{f}, \tilde{\boldsymbol{\rho}}, \tilde{\boldsymbol{\phi}}\} e^{i(\omega t+m \varphi-k z)} \\
& =\{\tilde{f}(r), \tilde{\boldsymbol{\rho}}(r), \tilde{\boldsymbol{\phi}}(r)\} e^{i(\omega t+m \varphi-k z)}
\end{aligned}
$$

the linearized Vlasov equation can be treated by integrating over the unperturbed particle orbit to yield

$$
\tilde{f}=\frac{2 q n_{0}}{\pi m_{b}}\left\{\delta\left(v_{z}-v_{o}\right) \tilde{\phi} \frac{d \delta_{\perp}}{d v_{\perp}^{2}}-\left[\frac{i k \delta_{\perp}}{2} \frac{d \delta\left(v_{z}-v_{o}\right)}{d v_{z}}+i\left(\omega-k v_{z}\right) \delta\left(v_{z}-v_{o}\right) \frac{d \delta_{\perp}}{d v_{\perp}^{2}}\right] \int_{0}^{\infty} \tilde{\phi}^{\prime} e^{i m\left(\varphi^{\prime}-\varphi\right)-i \Omega \tau} d \tau\right\},
$$

where $\delta_{\perp}=\delta\left[v_{\perp}^{2}-\nu^{2}\left(a^{2}-r^{2}\right)\right]$,

$$
\tau=t-t^{\prime}
$$

is the duration between the present time $t$ and the past time $t^{\prime}$, and the prime indicates the dependence on $\tau$ or $t^{\prime}$, e.g., $\tilde{\phi}^{\prime}=\tilde{\phi}\left[r^{\prime}(\tau)\right]$. Performing the Fourier transformation to Eq. (11) according to Eq. (12), making a substitution of Eq. (13), and applying Eqs. (7)-(9), we derive the following differential-integral equation:

$$
\begin{aligned}
\frac{1}{r} \frac{\partial}{\partial r}\left(r \frac{\partial \tilde{\phi}}{\partial r}\right)-\left(\frac{m^{2}}{r^{2}}+k^{2}\right) \tilde{\phi}= & \frac{\omega_{p}^{2}}{a \nu^{2}} \tilde{\phi}(a) \delta(r-a) \\
& +\frac{2 \omega_{p}^{2}}{\pi}\left[i \Omega \int_{-\infty}^{\infty} \int_{-\infty}^{\infty} d v_{r} d v_{\varphi} \frac{d \delta_{\perp}}{d v_{\perp}^{2}} \int_{0}^{\infty} \tilde{\phi}\left(r^{\prime}\right)\left(\frac{1}{r^{\prime}}\right)^{m}\left(\xi_{1}^{\prime} e^{i \theta}+\xi_{2}^{\prime}\right)^{m} e^{-i \Omega \tau} d \tau\right. \\
& \left.+\frac{k^{2}}{2} \int_{-\infty}^{\infty} \int_{-\infty}^{\infty} d v_{r} d v_{\varphi} \delta_{\perp} \int_{0}^{\infty} \tau \tilde{\phi}\left(r^{\prime}\right)\left(\frac{1}{r^{\prime}}\right)^{m}\left(\xi_{1}^{\prime} e^{i \theta}+\xi_{2}^{\prime}\right)^{m} e^{-i \Omega \tau} d \tau\right]
\end{aligned}
$$

where

$$
\Omega=\omega-k v_{o}
$$

is the Doppler-shifted frequency, and use has been made of the relations

$$
\int_{-\infty}^{\infty} \int_{-\infty}^{\infty} \tilde{\phi} \frac{d \delta_{\perp}}{d v_{\perp}^{2}} d v_{r} d v_{\varphi}=-\frac{\pi}{\nu^{2}} \tilde{\phi} \delta\left(r^{2}-a^{2}\right)
$$

and

$$
\begin{gathered}
\int_{-\infty}^{\infty} d v_{z} \frac{d \delta\left(v_{z}-v_{o}\right)}{d v_{z}} \int_{0}^{\infty} \tilde{\phi}^{\prime} e^{-i \Omega \tau} d \tau \\
=-i k \int_{0}^{\infty} \tau \tilde{\phi}^{\prime} e^{-i \Omega \tau} d \tau
\end{gathered}
$$

Solving Eq. (15) led to a dispersion relation which we will pursue in the next section.

\section{DISPERSION RELATION}

We consider the solution of Eq. (15) outside the beam first. Letting $\tilde{\phi}_{o}=\tilde{\phi}_{o}(r)$ be the perturbed electric potential outside the beam and $Z$ be the wall impedance in units of $Z_{0}=377 \Omega$ at the surface of the beam pipe, the boundary condition at $r=b$, in accordance with the electrostatic approximation, can be written as

$$
\tilde{\phi}_{o}(b)=\left.\frac{i \omega Z}{c k^{2}} \frac{d \tilde{\phi}_{o}}{d r}\right|_{r=b}
$$

This implies that the solution for $\tilde{\phi}_{o}(r)$ is 


$$
\begin{array}{r}
\tilde{\phi}_{o}(r)=\Lambda\left\{I_{m}(k r) K_{m}(k b)-I_{m}(k b) K_{m}(k r)\right. \\
-\left(\frac{i \omega Z}{c k^{2}}\right)\left[I_{m}(k r) \frac{d K_{m}(k b)}{d b}\right. \\
\left.\left.-K_{m}(k r) \frac{d I_{m}(k b)}{d b}\right]\right\},
\end{array}
$$

where $\Lambda$ is a constant, and $I_{n}(x)$ and $K_{n}(x)$ are the $n$th order modified Bessel functions of the first and the second kinds, respectively.

To find the solution of Eq. (15) inside the beam, we expand the perturbed electric potential as a sum of Jacobi polynomials $P_{l}^{(m, 0)}(x)$ according to

$$
\tilde{\phi}(r)=\left(\frac{r}{a}\right)^{m} \sum_{l=0}^{\infty} G_{l} P_{l}^{(m, 0)}\left(1-\frac{2 r^{2}}{a^{2}}\right),
$$

where $r \leq a$, and $m=0,1,2, \ldots$, denotes the azimuthal harmonic number. The choice of expanding $\tilde{\phi}$ in terms of Jacobi polynomials is suggested by the solutions for the special case of $k=0$ (see the discussion in the next section). The analysis then proceeds by substituting Eq. (21) into the left-hand side of Eq. (15) to derive

$$
\begin{aligned}
\frac{1}{r} \frac{\partial}{\partial r}\left(r \frac{\partial \tilde{\phi}}{\partial r}\right)-\left(\frac{m^{2}}{r^{2}}+k^{2}\right) \tilde{\phi}=-\left(\frac{r}{a}\right)^{m} \sum_{l=0}^{\infty}(l+m+1) & {\left[\frac{4(2 l+m+2) A_{l+1}}{a^{2}}\right.} \\
& \left.+k^{2}\left(\frac{G_{l+1}}{2 l+m+3}+\frac{G_{l}}{2 l+m+1}\right)\right] P_{l}^{(m, 1)}\left(1-\frac{2 r^{2}}{a^{2}}\right),
\end{aligned}
$$

where

$$
A_{l}=\sum_{j=l}^{\infty}(-1)^{l+j} G_{j}
$$

and use has been made of Eqs. (C1) and (C16) in Appendix C as well as the relation

$$
\sum_{l=1}^{\infty} \sum_{j=0}^{l-1} F_{l j}=\sum_{j=0}^{\infty} \sum_{l=j+1}^{\infty} F_{l j}=\sum_{l=0}^{\infty} \sum_{j=l+1}^{\infty} F_{j l},
$$

for any $F_{l j}$. Note that from Eq. (23) we have

$$
G_{l}=A_{l}+A_{l+1} .
$$

Next, substituting Eq. (21) into the right-hand side (RHS) of Eq. (15) and carrying out the lengthy calculation outlined in Appendix A yield

$$
\begin{aligned}
\text { RHS }= & \frac{\omega_{p}^{2}}{a \nu^{2}} \delta(r-a) \sum_{l=0}^{\infty}(-1)^{l} G_{l}\left[1-i \Omega \int_{0}^{\infty} e^{-i \Omega \tau} \cos ^{m}(\nu \tau) P_{l}^{(0, m)}(T) d \tau\right] \\
& -\frac{2 i \Omega \omega_{p}^{2}}{a^{2} \nu^{2}}\left(\frac{r}{a}\right)^{m} \sum_{l=1}^{\infty} G_{l} P_{j}^{(m, 1)}\left(1-\frac{2 r^{2}}{a^{2}}\right) \sum_{j=0}^{l-1}(-1)^{l+j}(m+j+1) \int_{0}^{\infty} e^{-i \Omega \tau} \cos ^{m}(\nu \tau)\left[P_{j}^{(0, m)}(T)-P_{j+1}^{(0, m)}(T)\right] d \tau \\
& +\omega_{p}^{2} k^{2}\left(\frac{r}{a}\right)^{m} \sum_{l=0}^{\infty} G_{l} P_{j}^{(m, 0)}\left(1-\frac{2 r^{2}}{a^{2}}\right) \int_{0}^{\infty} \tau e^{-i \Omega \tau} \cos ^{m}(\nu \tau) P_{l}^{(0, m)}(T) d \tau
\end{aligned}
$$

where

$$
T=\cos (2 \nu \tau)
$$

Substituting Eqs. (22) and (26) into Eq. (15) leads to a recursion relation for $A_{l}$ :

$$
W_{l} A_{l+1}+\left(W_{l}+W_{l-1}+U_{l}\right) A_{l}+W_{l-1} A_{l-1}=0,
$$

where $l=1,2,3, \ldots$,

$$
U_{l}=2(m+2 l)+\left(\frac{\omega_{p}}{\nu}\right)^{2} \alpha\left[B_{l-1}(m, \alpha)-B_{l}(m, \alpha)\right],
$$

$$
\begin{gathered}
W_{j}=\frac{a^{2} k^{2}}{2(m+2 j+1)}\left[1+\left(\frac{\omega_{p}}{\nu}\right)^{2} \frac{\partial}{\partial \alpha} B_{j}(m, \alpha)\right], \\
\alpha=\Omega / \nu=\left(\omega-k v_{o}\right) / \nu, \\
B_{j}(m, \alpha)=i \int_{0}^{\infty} e^{-i \alpha x} \cos ^{m} x P_{j}^{(0, m)}(\cos 2 x) d x,
\end{gathered}
$$

and $j=0,1,2,3, \ldots$, . In arriving at Eq. (28), we have applied Eqs. (23)-(25), (C1), and (C10) and the orthogonality of Jacobi polynomials, Eq. (C8). We notice that the integration in Eq. (32) can be carried out to give 


$$
\begin{aligned}
B_{l}(2 n, \alpha)=\frac{1}{\alpha} \sum_{j=0}^{l} \frac{(-1)^{l-j}(2 n+j+1)_{l}(2 n+2 j) !}{j !(l-j) !\left(2^{2}-\alpha^{2}\right) \cdots\left[(2 n+2 j)^{2}-\alpha^{2}\right]}\left\{1-\frac{\alpha^{2}}{2 !}-\frac{\alpha^{2}\left(2^{2}-\alpha^{2}\right)}{4 !}-\cdots\right. \\
\left.-\frac{\alpha^{2}\left(2^{2}-\alpha^{2}\right) \cdots\left[4(j+n-1)^{2}-\alpha^{2}\right]}{(2 j+2 n) !}\right\}
\end{aligned}
$$

and

$$
\begin{aligned}
& B_{l}(2 n+1, \alpha)=\alpha \sum_{j=0}^{l} \frac{(-1)^{l-j+1}(2 n+j+2)_{l}(2 n+2 j+1) !}{j !(l-j) !\left(1-\alpha^{2}\right)\left(3^{2}-\alpha^{2}\right) \cdots\left[(2 n+2 j+1)^{2}-\alpha^{2}\right]}\left\{1+\frac{1-\alpha^{2}}{3 !}+\frac{\left(1-\alpha^{2}\right)\left(3^{2}-\alpha^{2}\right)}{5 !}+\cdots\right. \\
& \left.+\frac{\left(1-\alpha^{2}\right)\left(3^{2}-\alpha^{2}\right) \cdots\left[(2 j+2 n-1)^{2}-\alpha^{2}\right]}{(2 j+2 n+1) !}\right\},
\end{aligned}
$$

where $n=0,1,2, \ldots,(x)_{j}=\Gamma(x+j) / \Gamma(x), \Gamma(y)$ is the Gamma function, and the imaginary part of $\omega$ is assumed to be negative. The recursion relation (28) reduces the number of unknowns in the expansion coefficients $A_{l}$ from infinity down to two, say $A_{0}$ and $A_{1}$. One should keep in mind that the quantities $A_{l}, U_{l}$, and $W_{l}$ are all functions of the harmonic number $m$. Also, since we are analyzing the beam stability in the linear regime, there should be no mixing of azimuthal mode numbers in Eq. (28).

Matching the electric potential inside the beam to the exterior solution $\tilde{\phi}_{o}(r)$ at $r=a$ yields

$$
\tilde{\phi}_{o}(a)=\tilde{\phi}(a)=A_{0},
$$

and

$$
\left.\frac{d \tilde{\phi}_{o}}{d r}\right|_{r=a}-\left.\frac{d \tilde{\phi}}{d r}\right|_{r=a}=\frac{\omega_{p}^{2}}{a \nu^{2}} \sum_{l=0}^{\infty}(-1)^{l} G_{l}\left[1-\alpha B_{l}(m, \alpha)\right] .
$$

Differentiating Eq. (21) and using Eqs. (C6), (C9), and (C10), we evaluate the derivative of $\tilde{\phi}$ at the surface of the beam as

$$
\left.\frac{d \tilde{\phi}}{d r}\right|_{r=a}=\frac{m}{a} A_{0}+\frac{2}{a} \sum_{l=0}^{\infty}(-1)^{l+1} l(m+l+1) G_{l} .
$$

Adding Eqs. (36) and (37), dividing the result by Eq. (35), and then applying Eqs. (23), (25), and (28), we derive the dispersion relation

$$
\left.\frac{a}{\tilde{\phi}_{o}(a)} \frac{d \tilde{\phi}_{o}}{d r}\right|_{r=a}-m-\left(\frac{\omega_{p}}{\nu}\right)^{2}\left[1-\alpha B_{0}(m, \alpha)\right]=W_{0}\left(1+\frac{A_{1}}{A_{0}}\right),
$$

where the ratio $A_{1} / A_{0}$ can be expressed in terms of infinite determinants or a continuous fractions as

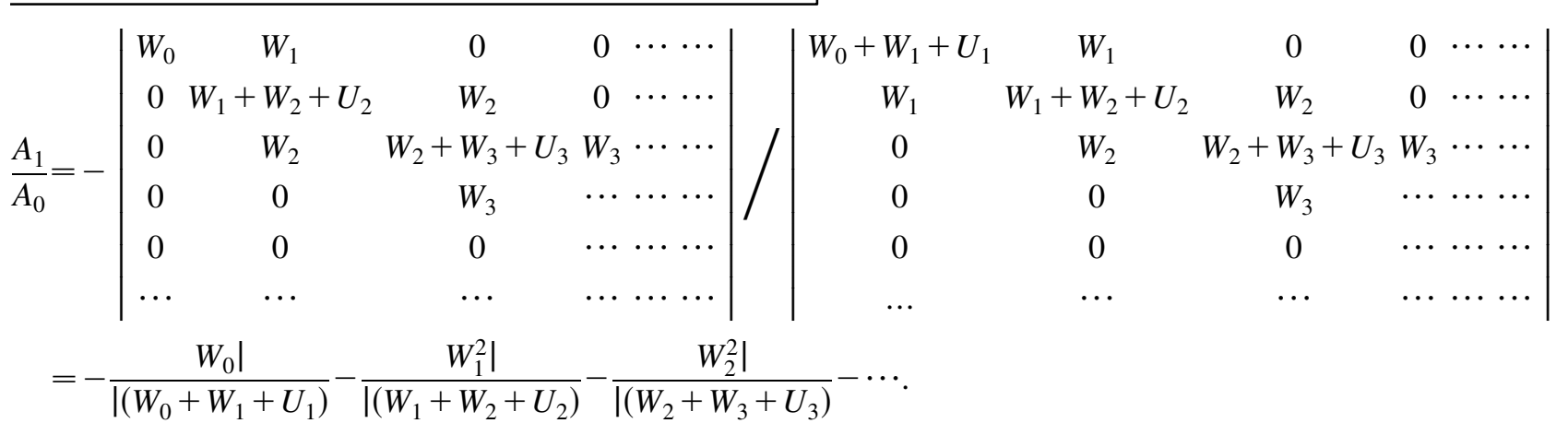

In order to perform a practical numerical computation, it is necessary to truncate the determinants in Eq. (39) at a certain finite rank.

\section{SPECIAL CASES}

\section{A. Axisymmetric $(m=0)$ perturbations} [12]

$$
\begin{gathered}
U_{l}=4 l+\left(\frac{\omega_{p}}{\nu}\right)^{2} \alpha\left[B_{l-1}-B_{l}(\alpha)\right], \\
W_{l}=\frac{a^{2} k^{2}}{2(2 l+1)}\left[1+\left(\frac{\omega_{p}}{\nu}\right)^{2} \frac{\partial}{\partial \alpha} B_{l}(\alpha)\right],
\end{gathered}
$$

and the dispersion relation (38) becomes

$$
\left.\frac{a}{\tilde{\phi}_{o}(a)} \frac{d \tilde{\phi}_{o}}{d r}\right|_{r=a}=W_{0}\left(1+\frac{A_{1}}{A_{0}}\right),
$$


where

$$
B_{l}(\alpha)=B_{l}(0, \alpha)=i \int_{0}^{\infty} e^{-i \alpha x} P_{l}(\cos 2 x) d x
$$

$P_{l}(x)=P_{l}^{(0,0)}(x)$ is the Legendre polynomial, and $A_{1} / A_{0}$ is given in Eq. (39). Readers are referred to Ref. [12] for detailed discussions on the axisymmetric modes.

\section{B. Axially uniform $(k=0)$ modes}

This is the case studied previously by Gluckstern [8]. Here, we will derive Gluckstern's results by setting $k=0$ in our solution.

When $k=0$, we find $W_{l}=0$ and the recursion relation (28) is reduced to

$$
U_{j} A_{j}=0,
$$

for $j \neq 0$ and $j=1,2,3, \ldots$. The nontrivial solutions are $A_{0}=0$, and $U_{j}=0$ when $A_{j} \neq 0$. In the case where only one of $A_{j}$ s is nonzero, we have $G_{j-1}=G_{j}=A_{j}$ and the Vlasov-Poisson equations admit the eigenfunction

$$
\tilde{\phi}_{j}(r)=A_{j}\left(\frac{r}{a}\right)^{m}\left[P_{j}^{(m, 0)}\left(1-\frac{2 r^{2}}{a^{2}}\right)+P_{j-1}^{(m, 0)}\left(1-\frac{2 r^{2}}{a^{2}}\right)\right]
$$

with $U_{j}=0$ as the corresponding eigenvalue equation. This solution implies $\tilde{\phi}(a)=\tilde{\phi}_{o}(r)=0$, i.e., the perturbed field vanishes outside the beam. Further, the perturbed field is nonzero at the center of the beam only for $m=0$. We notice that for $k=0$, one has $\alpha=\Omega / \nu=$ $\omega / \nu$ and $B_{l}(m, \alpha)=B_{l}(m, \omega / \nu)$. Then, using Eqs. (29), (C9), and (C11) we can show that the eigenfunction given in Eq. (45) and the eigenvalue equation $U_{j}=0$ (the dispersion relation) can be expressed in terms of the hypergeometric function ${ }_{2} F_{1}(a, b ; c ; z)$ as

$$
\begin{aligned}
\tilde{\phi}_{j}(r)= & A_{j} \frac{(m+2 j)(m+j-1) !}{m ! j !}\left(\frac{r}{a}\right)^{m} \\
& \times{ }_{2} F_{1}\left(-j, m+j ; m+1 ; r^{2} / a^{2}\right),
\end{aligned}
$$

and

$$
\begin{aligned}
& \int_{0}^{\infty} e^{-i(\omega / \nu) x} \cos ^{m} x_{2} F_{1}\left(-j, m+j ; m+1 ; \cos ^{2} x\right) d x \\
& \quad=\frac{2 \nu}{i \omega}\left[\frac{(-1)^{j} m ! j ! \nu^{2}}{(m+j-1) ! \omega_{p}^{2}}\right],
\end{aligned}
$$

respectively, for $j=1,2,3, \ldots$, and $m=0,1,2,3, \ldots$, . Equations (46) and (47) were obtained previously by Gluckstern via a series solution approach.

Now consider the solution for $k=0, j=0$, and $m \neq 0$. The recursion relation (28) then admits the solutions $G_{0}=A_{0} \neq 0$ and $G_{l}=A_{l}=0$ for $l \neq 0$. Hence, differing from the $j \neq 0$ modes, the perturbed field does not vanish outside the beam in this case. Applying the boundary conditions (35) and (36) together with the expansion in Eq. (21), we derive the following dispersion relation for the particular case of $k=0, j=0$, and $m \neq 0$ :

$$
\left.\frac{a}{\tilde{\phi}_{o}(a)} \frac{d \tilde{\phi}_{o}}{d r}\right|_{r=a}=m+\left(\frac{\omega_{p}}{\nu}\right)^{2}\left[1-\left(\frac{\omega}{\nu}\right) B_{0}(m, \omega / \nu)\right] .
$$

If the outer boundary is at $r=\infty$, and the boundary condition is $\tilde{\phi}_{o}(r \rightarrow \infty)=0$, then the solution of the Poisson equation in the region outside the beam is

$$
\tilde{\phi}_{o}(r)=A_{0}\left(\frac{a}{r}\right)^{m} .
$$

The dispersion relation (48) is then simplified to

$$
\int_{0}^{\infty} e^{-i \omega x / \nu} \cos ^{m} x d x=\frac{\nu}{i \omega}\left(1+\frac{2 m \nu^{2}}{\omega_{p}^{2}}\right) .
$$

Since $P_{0}^{(m, 0)}(x)=1$, the solution for the perturbed field inside the beam is given by

$$
\tilde{\phi}(r)=A_{0}\left(\frac{r}{a}\right)^{m} .
$$

The perturbed charge density is nonzero only on the surface of the beam. Equations (49)-(51) were also derived by Gluckstern. Readers are referred to Ref. [8] for more discussions of the axially uniform modes. For a beam propagating inside a perfect conducting pipe of radius $b$, the external solution is

$$
\tilde{\phi}_{o}(r)=A_{o}\left(\frac{a}{r}\right)^{m}\left[1-\left(\frac{r}{b}\right)^{2 m}\right],
$$

and the dispersion relation (48) can be rewritten as

$$
\begin{aligned}
B_{0}(m, \omega / \nu) & =i \int_{0}^{\infty} e^{-i \omega x / \nu} \cos ^{m} x d x \\
& =\frac{\nu}{\omega}\left\{1+\frac{2 m \nu^{2}}{\omega_{p}^{2}\left[1-(a / b)^{2 m}\right]}\right\} .
\end{aligned}
$$

\section{Cold-beam $(\nu=0)$ limit}

The model we are studying approaches the cold-beam limit when $\nu \rightarrow 0$. To explore this special case, we first consider some limits when $\nu$ approaches 0. From Eqs. (33) and (34) we find

$$
\lim _{\nu \rightarrow 0} \alpha B_{l}(m, \alpha)=1
$$

and

$$
\lim _{\nu \rightarrow 0} \frac{\partial}{\partial \alpha} B_{l}(m, \alpha)=-\lim _{\nu \rightarrow 0}\left(\frac{1}{\alpha^{2}}\right),
$$

for $m=0,1,2, \ldots$, where use has been made of Eq. (C10). Then, from Eq. (54) we know that when $\nu \rightarrow 0$, the limit of $\alpha\left[B_{l-1}(m, \alpha)-B_{l}(m, \alpha)\right] / \nu^{2}$ approaches that of $\partial\left[\alpha B_{l-1}(m, \alpha)-\alpha B_{l}(m, \alpha)\right] / \partial \nu^{2}$. Therefore, using the relation 


$$
\lim _{\nu \rightarrow 0} \frac{\partial}{\partial \nu^{2}}\left[\alpha B_{l}(m, \alpha)\right]=\frac{1}{\Omega^{2}}[m+2 l(l+m+1)],
$$

we find

$$
\lim _{\nu \rightarrow 0}\left\{\alpha\left[B_{l-1}(m, \alpha)-B_{l}(m, \alpha)\right] / \nu^{2}\right\}=\frac{-2(2 l+m)}{\Omega^{2}}
$$

Next, we discuss the limits of $U_{l}, V_{l}$, and Eq. (28) when $\nu \rightarrow 0$. Using Eqs. (28)-(30), (55), and (57), one can show that when $\nu=0$,

$$
\begin{gathered}
U_{l}=2(2 l+m)\left[1-\left(\frac{\omega_{p}}{\Omega}\right)^{2}\right], \\
W_{l}=\frac{a^{2} k^{2}}{2(m+2 l+1)}\left[1-\left(\frac{\omega_{p}}{\Omega}\right)^{2}\right],
\end{gathered}
$$

and

$$
\begin{aligned}
\frac{(a k / 2)^{2}}{m+2 l+1} A_{l+1} & +\left[\frac{(a k / 2)^{2}}{m+2 l+1}+\frac{(a k / 2)^{2}}{m+2 l-1}+m+2 l\right] A_{l} \\
& +\frac{(a k / 2)^{2}}{m+2 l-1} A_{l-1}=0
\end{aligned}
$$

Comparing Eq. (60) with the recursion relation of Bessel functions, we infer that

$$
A_{l}=h(-1)^{l} I_{2 l+m}(a k),
$$

where $h$ is a constant. Using Eqs. (54), (59), and (61), one can show that the dispersion relation (38) can be rewritten as

$$
\left.\frac{a}{\tilde{\phi}_{o}(a)} \frac{d \tilde{\phi}_{o}}{d r}\right|_{r=a}=\left[1-\left(\frac{\omega_{p}}{\Omega}\right)^{2}\right]\left[m+\frac{a k I_{m+1}(a k)}{I_{m}(a k)}\right] .
$$

This is the familiar dispersion relation for a cold-beam [39,40]. Applying Eqs. (21), (25), (61), and (C18), one can also show that the cold-beam limit for the electric potential inside the beam is given by $\tilde{\phi}(r) \propto I_{m}(k r)$ - another familiar result.

\section{Long-wavelength surface perturbations}

In the long-wavelength $(0<k a<k b \ll 1)$ region, we expect that many characteristics of the pure transverse modes described in Eqs. (44) and (48) are not altered very much by the longitudinal perturbation. Here, we narrow our concentration on the $m=1$ and 2 harmonics (the dipole and the quadrupole modes) for the surface perturbations, the modes associated with the ones described in Eq. (48), in the long-wavelength region. It should be noted here that although the perturbed charge density peaks near the beam surface, the wave we are studying here is different from the "surface wave" in a neutral-plasma column [41]. Our purpose is to compare our dispersion relation of the dipole and the quadrupole modes in the long-wavelength region with those derived from using the approximation of no radial-density fluctuation inside the beam and the approximation of neglecting the longitudinal electric field due to the perturbed charge distribution $[4-6,10]$. Thus, neglecting the right-hand side of Eq. (38) that contains the information of radial modes with $j \geq 1$, we obtain

$$
\left.\frac{a}{\tilde{\phi}_{o}(a)} \frac{d \tilde{\phi}_{o}}{d r}\right|_{r=a}-m-\left(\frac{\omega_{p}}{\nu}\right)^{2}\left[1-\alpha B_{0}(m, \alpha)\right]=0 .
$$

Equation (63) is almost identical to Eq. (48) except that $\alpha$ takes the place of $\omega / \nu$. It has two roots for $\Omega$ when $m=1$ or 2. For $m>2$, Eq. (63) has more than two roots. Substituting Eq. (20) into Eq. (63) and applying the small argument expansions of Bessel functions yields

$$
\frac{\nu^{2}}{\alpha B_{0}(m, \alpha)-1} \approx \frac{\frac{\omega_{p}^{2}}{2 m}\left[1-\frac{a^{2 m}}{b^{2 m}}-\frac{i m Z}{k b}\left(1+\frac{a^{2 m}}{b^{2 m}}\right)\right]}{1-i m Z /(k b)},
$$

where

$$
Z=\frac{\omega Z}{c k} \text {. }
$$

When $Z=0$ and $k=0$, Eq. (64) reduces to Eq. (53).

For the dipole mode, we make the substitution $m=1$ in Eq. (64) and use Eq. (34) to derive

$$
\left(\omega-k v_{o}\right)^{2}-\nu^{2} \approx \frac{\frac{\omega_{p}^{2}}{2}\left[1-\frac{a^{2}}{b^{2}}-\frac{i Z}{k b}\left(1+\frac{a^{2}}{b^{2}}\right)\right]}{1-i Z /(k b)} .
$$

For $Z=0$, Eq. (66) becomes the customary dispersion relation of the dipole mode for a continuous nonrelativistic beam having zero axial momentum spread in a perfect conducting pipe. For the quadrupole mode, taking $m=2$ and substituting Eq. (33) into Eq. (64), we have

$$
\left(\omega-k v_{o}\right)^{2}-4 \nu^{2} \approx \frac{\frac{\omega_{p}^{2}}{2}\left[1-\frac{a^{4}}{b^{4}}-\frac{2 i Z}{k b}\left(1+\frac{a^{4}}{b^{4}}\right)\right]}{1-2 i Z /(k b)}
$$

The dispersion relations given in Eqs. (66) and (67) are similar to the dispersion relations for the dipole and the quadrupole modes derived previously $[4-6,10]$. In the following, the modes described in Eq. (63) will be referred to as the usual transverse modes.

\section{E. Low-frequency coupling modes}

As will be discussed more in the next section, our three-dimensional analysis reveals two new classes of coupling modes originated from the interaction between longitudinal and transverse degrees of motion. It is difficult to explore the coupling modes in general via analytical calculation. However, in the limited parameter range considered below, it is possible to find the approximate analytical solutions for the "low-frequency coupling modes." We proceed with the discussion by considering the special case of $l=2 n(n=0,1,2,3, \ldots$,$) with m$ and 
$l$ are not simultaneously equal to zero in the recursion relation (28). We assume the expansion coefficients $A_{l}$ are normalized in such a way that the magnitude of $A_{l}$ and $A_{l+1}$ is about 1 and $\left|A_{i}\right| \ll 1$ for $i<l$ or $i>l+1$. Let us concentrate on the regime of $(a k)^{2}<2(m+4 n+3)$, $\alpha^{2} \ll 1$, and $\omega_{p}^{2} \ll \nu^{2}$. Using the limits

$$
\begin{gathered}
\lim _{\alpha \rightarrow 0} \alpha B_{2 i+1}(2 j, \alpha)=\lim _{\alpha \rightarrow 0} \alpha B_{n}(2 j+1, \alpha)=0, \\
\lim _{\alpha \rightarrow 0} \alpha^{2} \frac{d}{d \alpha} B_{2 n+1}(j, \alpha)=0,
\end{gathered}
$$

and

$$
\begin{aligned}
\lim _{\alpha \rightarrow 0} \alpha^{2} \frac{d}{d \alpha} B_{2 n}(2 j, \alpha) & =-\bar{P}_{2 n}^{(0,2 j)} \\
& =\frac{-1}{2 \pi} \int_{0}^{2 \pi} \cos ^{2 j} t P_{2 n}^{(0,2 j)}(\cos 2 t) d t \\
& =\sum_{i=0}^{2 n} \frac{(-1)^{i+1}(2 j+i+1)_{2 n}(2 j+2 i) !}{i !(2 n-i) ! \cdot 2^{2} \cdot 4^{2} \cdots(2 j+2 i)^{2}},
\end{aligned}
$$

for zero and positive integers $i, j$, and $n$, we find that

$$
\begin{gathered}
\lim _{\alpha \rightarrow 0} U_{l}=2(m+2 l), \\
\lim _{\alpha \rightarrow 0} W_{2 n}=\frac{-\left(k a \omega_{p}\right)^{2} \bar{P}_{2 n}^{(0,2 j)}}{2(2 j+4 n+1) \Omega^{2}},
\end{gathered}
$$

and

$$
\lim _{\alpha \rightarrow 0} W_{2 n+1}=\frac{(k a)^{2}}{2(m+4 n+3)} \ll 1 .
$$

Then from the recursion relation, Eq. (28), we can derive

$$
W_{l} A_{l+1}+\left(U_{l}+W_{l}\right) A_{l} \approx 0
$$

and

$$
\left(U_{l+1}+W_{l}\right) A_{l+1}+W_{l} A_{l} \approx 0 .
$$

For nonzero $A_{l}$ and $A_{l+1}$, one has the approximate solution

$$
W_{l} \approx \frac{-U_{l} U_{l+1}}{U_{l}+U_{l+1}},
$$

for Eqs. (74) and (75). Substituting the limits in Eqs. (71)(73) into Eq. (76) yields

$$
\Omega^{2} \approx \frac{\left(k a \omega_{p}\right)^{2} \bar{P}_{l}^{(0, m)}}{2(m+2 l)(m+2 l+2)},
$$

where $l$ and $m$ are non-negative even integers and are not simultaneously equal to zero. Equation (77) gives the approximate $\Omega^{2}$ of the low-frequency coupling modes in the parameter range described earlier. The result in Eq. (77) indicates that the value of $\Omega$ vanishes at zero beam intensity, i.e., $\Omega \rightarrow 0$ when $\omega_{p} \rightarrow 0$. Using either Eq. (74) or (75), we can show that

$$
A_{l+1} \approx \frac{U_{l} A_{l}}{U_{l+1}} \approx\left(\frac{m+2 l}{m+2 l+2}\right) A_{l}
$$

at the frequency given in Eq. (77). For this kind of modes, when $l>0$, the perturbed potential almost vanishes at the edge of the beam. We note that perturbations of odd azimuthal harmonics do not have the low-frequency coupling modes.

\section{NUMERICAL RESULTS}

The discussion in this section is based on the numerical solution of Eq. (38). As mentioned earlier, the infinite determinants in Eq. (39) have to be truncated at a certain finite rank for a practical numerical computation. Such an approach has been proven to give reasonably good results $[12,15,16]$. When finite-rank determinants are used in Eq. (38), the dispersion relation is then reduced to a finite-order algebraic equation and hence the number of roots or modes can be counted. The rank of truncation is mainly determined by the number of modes to be examined and the desired accuracy. We notice that when $k=0$, only the transverse modes described by $U_{j}=0$ exist. Therefore, if one wishes to study the modes contained in $U_{n}=0$, then the truncated determinants have to include $U_{n}$. That is, one has to truncate the infinite determinants at rank of at least $n$. However, the relation between the accuracy of root computation and the rank of truncation is much less apparent. In principle, one could truncate the determinants at a rank much higher than $n$ to achieve better accuracy in computing the roots. But, the bigger the determinants used in the computation, the larger the number of roots that will be found and the smaller the separations between roots, so that discerning of modes becomes more complicated. In practice, the identification of modes turns tedious when the infinite determinants are truncated to any rank higher than three for root computation. The approaches adopted to identify modes are (a) comparing the solutions between the $k>0$ and the $k=0$ cases, (b) comparing the solutions among different ranks of truncation, and (c) checking the radial mode structure. Since the roots, and hence the radial mode structure can not be very accurately computed by using finite determinants, the first two approaches are the primary methods used.

The roots of the dispersion relation (38) fall into three classes: (i) the $T$ modes are the ones that approach the solutions of $U_{j}=0$ (the pure transverse modes) when $k \rightarrow 0$, (ii) the "high-frequency coupling modes" having the limit of $\Omega \rightarrow n \nu_{o}>0$ when $\omega_{p} \rightarrow 0$, and (iii) the low-frequency coupling modes with $\Omega \rightarrow 0$ when $\omega_{p} \rightarrow$ 0 as discussed in Sec. IV E. Both types of coupling modes are due to the coupling between longitudinal and transverse motions and therefore vanish when $k=0$ or when the betatron motion is neglected or when longitudinal and transverse perturbations are treated separately. The highfrequency coupling modes do not exist in the axisymmetric perturbations. The low-frequency coupling modes exist only in the perturbations of even and zero azimuthal 
harmonics. The usual transverse modes found in the customary analyses [4-7] correspond to density perturbations on the beam surface and hence are similar to the lowest radial modes in class (i) as discussed earlier in Sec. IV D. In the study here, we shall concentrate on some low radial modes, i.e., the modes found in the dispersion relation with the infinite determinants truncated at low ranks. Hence for simplicity, when there is no strong necessity to make the distinction, we shall use the notation $T_{m, j}(j>0)$ to represent the whole family of $T$ modes in the $m$ th azimuthal harmonic which are related to $U_{j}=$ 0 when $k \rightarrow 0$. The usual transverse modes in Eq. (63) will be referred to as $T_{m, 0}$ modes and the high-frequency coupling modes will be designated as $C_{m, j}$ modes. The low-frequency coupling modes will be referred to as $L_{m, n}$ modes for $n \geq 1$, in the order of their first appearance in the dispersion relation using $(2 n-1) \times(2 n-1)$ determinants for approximation.

There are five parameters involved in the dispersion relation: the azimuthal harmonic number $m$, the ratio between the radii of the surrounding pipe and the beam $b / a$, the axial wavelength parameter $k a$, the tune depression $\nu / \nu_{o}$, and the wall impedance parameter $Z=$ $\omega Z /(c k)$. We limit our numerical studies here to the azimuthal harmonics $m=1$ and 2 (the dipole and the quadrupole modes). Readers should consult Ref. [12] for the numerical results for the $m=0$ modes. To narrow the parameter space further, we consider only the cases of $k a=0.0$ and 2.0 for $b / a=1.5$. The wall impedances investigated are of perfect conductor and resistive types with $Z=0.2$. For each one of the eight combinations of these five parameter values, we have computed $\Omega / \nu_{o}$, for the full range of tune depression from $\nu / \nu_{o}=0$ to $\nu / \nu_{o}=1$. The radial structures of the modes were estimated using the approximation

$$
\begin{gathered}
\tilde{\phi}(r) \sim\left(\frac{r}{a}\right)^{m}\left\{1+\sum_{l=1}^{n}\left(\frac{A_{l}}{A_{0}}\right)\left[P_{l-1}^{(m, 0)}\left(1-\frac{2 r^{2}}{a^{2}}\right)\right.\right. \\
\left.\left.+P_{l}^{(m, 0)}\left(1-\frac{2 r^{2}}{a^{2}}\right)\right]\right\},
\end{gathered}
$$

derived from Eqs. (21) and (25). For a given tune depression, the expansion coefficients $A_{l} / A_{0}$ here were computed by using the recursion relation (28) and by substituting the computed mode frequency into Eq. (38). Because the value of roots cannot be precisely computed by using determinants of finite rank, and iterating the recursion relation augments errors, the coefficient $A_{l} / A_{0}$, and hence the radial mode structure, cannot be accurately estimated, especially when $l$ is greater than the rank of truncation. Nonetheless, we will present some examples to give readers a crude picture of the radial modes. The numerical results for the cases of $m=1$ and 2 will be discussed separately in the following.

\section{A. Dipole modes $(m=1)$}

In the case of $m=1$, if the determinants in Eq. (39) are truncated at rank $n(n>1)$, one obtains $(n+1)^{2}$ roots for $\alpha^{2}$. Among these roots, $n(n+3) / 2$ originate from the solutions of $U_{j}=0$ for $j=1,2, \ldots, n$, including one root for the mode discussed in Eq. (63), and the remaining $\left(n^{2}+n+2\right) / 2$ roots belong to the class of high-frequency coupling modes. When $k=0$, only the $n(n+3) / 2$ roots associated with the pure transverse modes are nontrivial solutions. We limit our study to the first sixteen $m=1$ modes, i.e., up to the $T_{1,3}$ modes, obtainable from truncating the determinants at a rank equal to or higher than 3 . We choose to use $4 \times 4$ determinants for improved accuracy and easy identification of modes. The solutions of the dispersion relation then also include nine roots associated with $T_{1,4}$ and $C_{1,4}$ modes. These roots are ignored because of poor accuracy. For zero wall impedance, the absolute value of the real part of $\Omega / \nu_{o},\left|\operatorname{Re}\left(\Omega / \nu_{o}\right)\right|$, is depicted in Figs. 1 and 2 as a function of tune depression $\nu / \nu_{o}$ for the cases of $k=0$ and $a k=2$, respectively. The absolute value of the corresponding imaginary part of $\Omega / \nu_{o},\left|\operatorname{Im}\left(\Omega / \nu_{o}\right)\right|$, is shown in Figs. 3 and 4 where only the modes with nonzero $\operatorname{Im}\left(\Omega / \nu_{o}\right)$ are displayed. Also shown in Fig. 4 is the $\left|\operatorname{Im}\left(\Omega / \nu_{o}\right)\right|$ of $T_{1,0}$ mode for the wall impedance of $Z=$ 0.2 . Figure 5 displays the detail of the real part of $\Omega^{2} / \nu_{o}^{2}$ as a function of $\nu / \nu_{o}$ in the high-intensity region for the case of $a k=2$.

As shown in Figs. 1 and 3, that for all modes, the values of $\left|\operatorname{Re}\left(\Omega / \nu_{o}\right)\right|$ start from the solutions of $U_{j}=0(j=1$, 2 , and 3), that is, from $1,3,5$, and 7 , at $\nu=\nu_{o}$, and

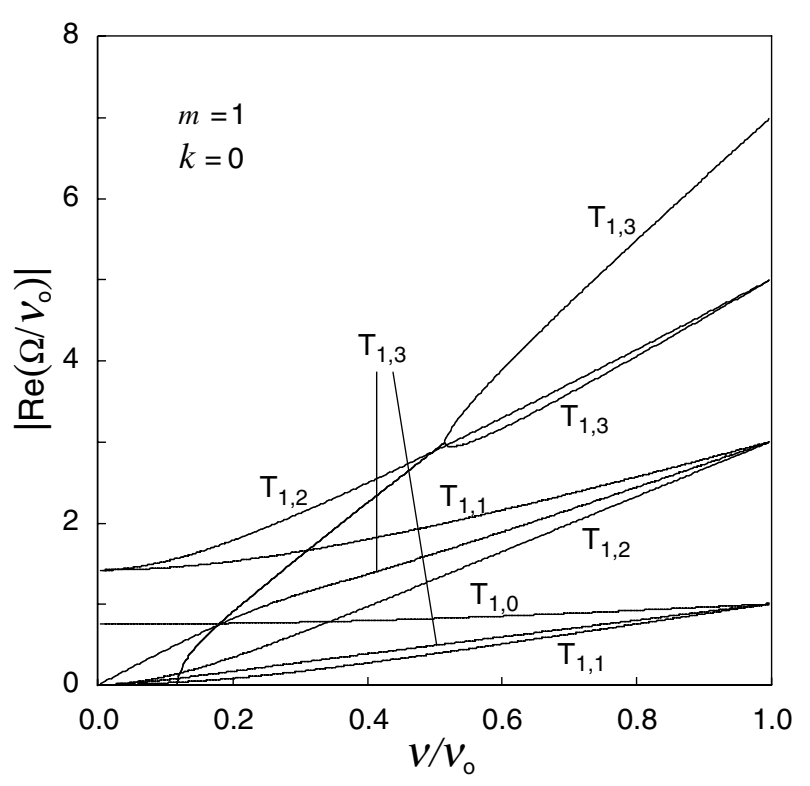

FIG. 1. Absolute value of the real part of $\Omega / \nu_{o}$ for the first nine dipole modes as a function of $\nu / \nu_{o}$ for $k=0, b / a=1.5$, and zero wall impedance $(Z=0)$. 


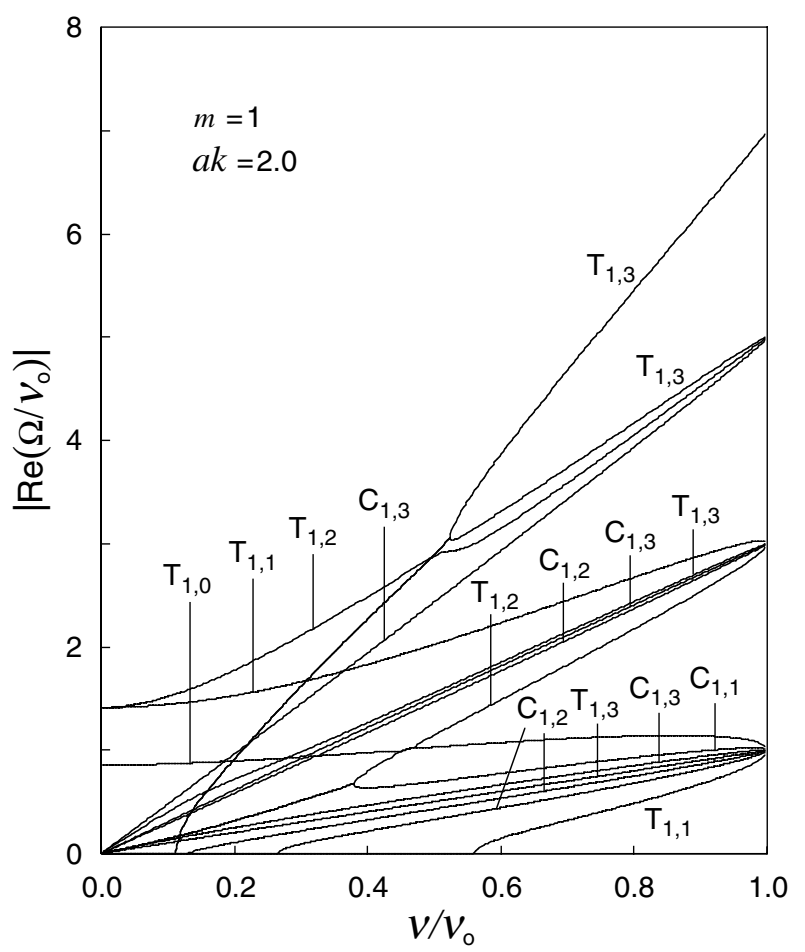

FIG. 2. Absolute value of the real part of $\Omega / \nu_{o}$ for the first 16 dipole modes as a function of $\nu / \nu_{o}$ for $a k=2.0, b / a=1.5$, and zero wall impedance $(Z=0)$.

decrease when the beam intensity increases. When $\nu \rightarrow 0$, the $T_{1,0}$ mode approaches the cold-beam limit, while the $\left|\operatorname{Re}\left(\Omega / \nu_{o}\right)\right|$ of the upper $T_{1,2}$ and $T_{1,1}$ modes end near 1.4. In the case of $k=0$, the Doppler-shifted frequency of all other modes, except for the highest $T_{1,3}$ modes, decreases to zero at $\nu=0$. A kind of obvious mode interaction appears in the high-intensity region as confluences of

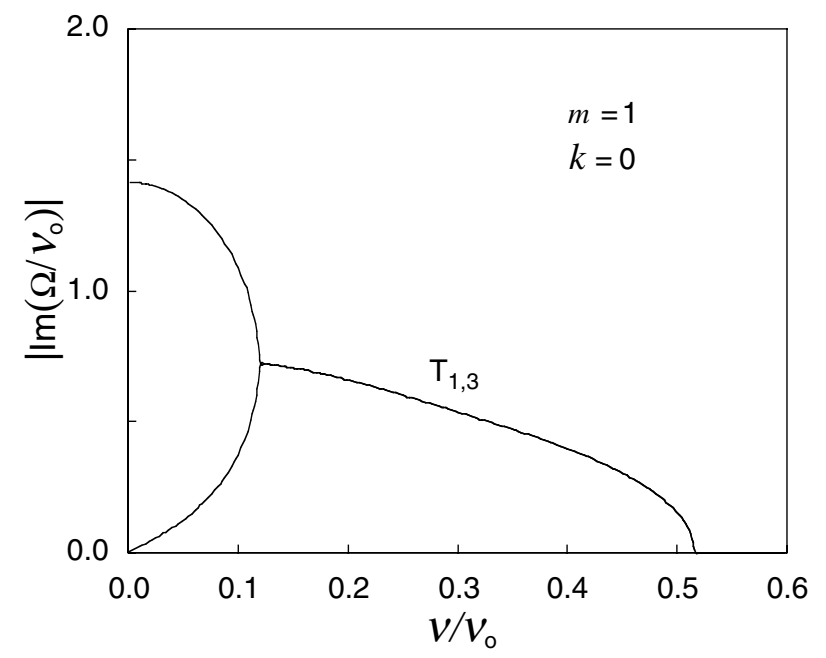

FIG. 3. Absolute value of the imaginary part of $\Omega / \nu_{o}$ for the first nine dipole modes as a function of $\nu / \nu_{o}$ for $k=0$ and $b / a=1.5$. Here, only the modes with none-zero $\operatorname{Im}\left(\Omega / \nu_{o}\right)$ are labeled.

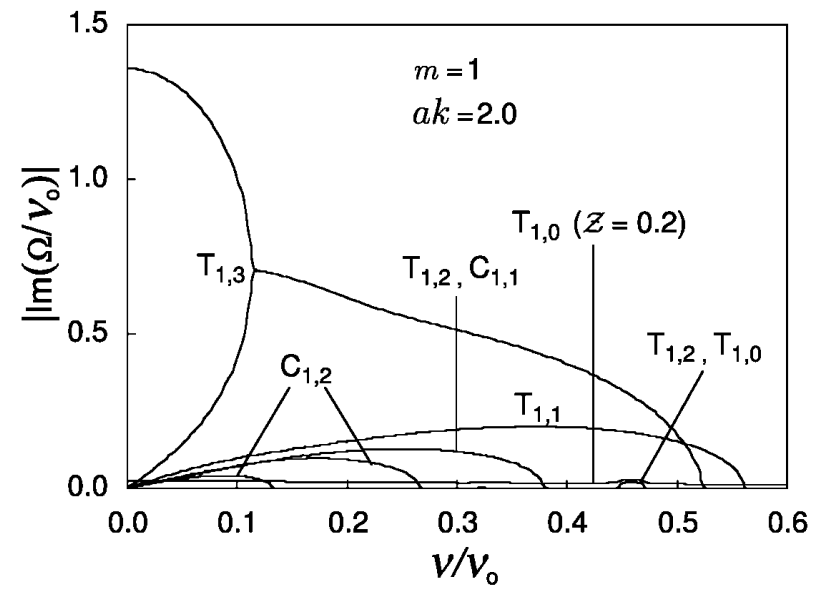

FIG. 4. Absolute value of the imaginary part of $\Omega / \nu_{o}$ for the first 16 dipole modes as a function of $\nu / \nu_{o}$ for $a k=2.0, b / a=$ 1.5 , and zero wall impedance. Only the modes with none-zero $\operatorname{Im}\left(\Omega / \nu_{o}\right)$ are labeled. Also shown is the absolute value of the imaginary part of $\Omega / \nu_{o}$ of the $T_{1,0}$ mode for the case of $Z=0.2$.

modes where two or more modes have the same real part of $\Omega$. The frequencies in the confluence regions are complex conjugate pairs indicating possible instability. A general characteristic of mode confluence is that both the span of the confluence region and the maximum growth rates in the region increase with increasing $k$. For $k=0$, a confluence occurs between the two upper $T_{1,3}$ modes in the region of $0.115 \leq \nu / \nu_{o} \leq 0.515$. The highest growth rate in this region, about $0.72 \nu_{o}$, occurs at the higherintensity end of the confluence. The real part of the $\Omega$ of these two modes falls to zero near $\nu=0.112 \nu_{o}$. The highest $T_{1,3}$ modes have the largest growth rate $(\approx$ $1.36 \nu_{o}$ at $\nu=0$ ) among all the dipole modes studied.

In the case of $a k=2$, two more confluences emerge: the confluence of $T_{1,0}$ and $T_{1,2}$ near $\nu=0.46 \nu_{o}$, and the

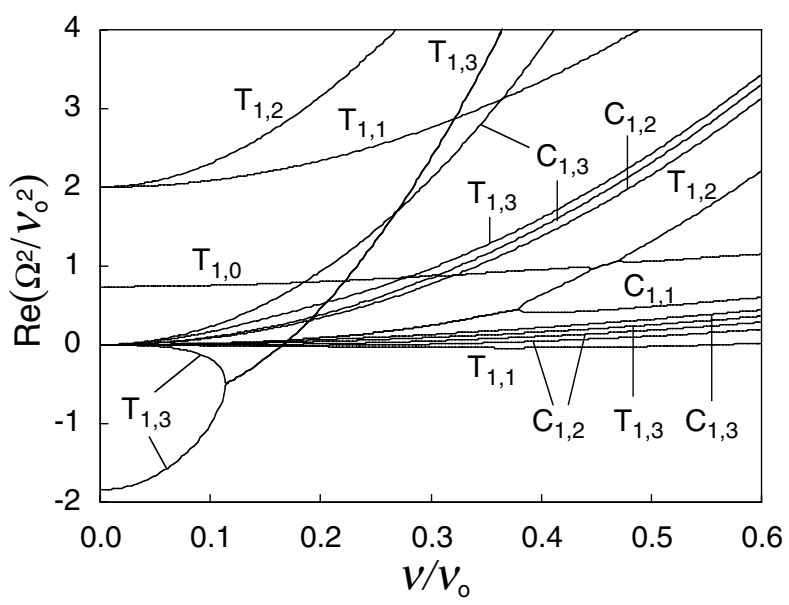

FIG. 5. The real part of $\Omega^{2} / \nu_{o}^{2}$ for the first 16 dipole modes as a function of $\nu / \nu_{o}$ for $a k=2.0, b / a=1.5$, and zero wall impedance. 
confluence of $C_{1,1}$ and $T_{1,2}$ between $\nu=0$ and $\nu=$ $0.38 \nu_{o}$. Although the confluence of $T_{1,0}$ and $T_{1,2}$ has a relatively small growth rate of $0.02 \nu_{o}$, it demonstrates that two originally independent, stable transverse modes can interact through longitudinal perturbation to become unstable. The lower $T_{1,1}$ mode is the one among all the $T$ modes affected most by the longitudinal perturbation. For $a k=2$, it becomes unstable with a maximum growth rate around $0.2 \nu_{o}$ when $\nu \approx 0.37 \nu_{o}$, and the real part of its Dopper-shifted frequency falls to zero near $\nu=$ $0.56 \nu_{o}$. The frequencies of all $T_{1,3}$ modes are very close to those in the $k=0$ case indicating that these modes are not sensitive to the longitudinal perturbation. When $k \neq$ 0 , only the $T_{1,0}$ mode and those highest $T$ modes, i.e., the highest $T_{1,1}, T_{1,2}$, and $T_{1,3}$, have nonzero $\Omega$ when $\nu=0$. For $a k=2$, the $\operatorname{Re}\left(\Omega^{2} / \nu_{o}^{2}\right)$ of the two lower $C_{1,2}$ drop to below zero separately in the regions of $\nu<0.133 \nu_{o}$ and $\nu<0.267 \nu_{o}$ indicating these two modes are unstable in the high-intensity regime as shown in Figs. 4 and 5. We note that the highest growth rate found in dipole modes is substantially higher than that of the axisymmetric $T_{3}$ mode $\left(\approx 0.21 \nu_{o}\right)$ studied in Ref. [12].

Intuitively, we expect that only the mode(s) with appreciable field intensity outside the beam can be influenced by the wall impedance. We investigated the effect of resistive wall impedance and found that only the usual dipole mode, the $T_{1,0}$ mode, is appreciably affected by the resistive wall impedance. The highest growth rate occurs near $\nu \approx 0$. For $Z=0.2$, the maximum growth rate of the $T_{1,0}$ mode is about $0.023 \nu_{o}$ as shown in Fig. 4. The approximate radial structures of the modes studied are depicted in Fig. 6 where the results were computed by using the expansion coefficients up to $A_{4}$ for $a k=2.0$, $\nu=0.697 \nu_{o}$, and zero wall impedance.

\section{B. Quadrupole modes $(m=2)$}

For $m=2$, if determinants of $n$th rank are employed in Eq. (38) to solve for $\alpha^{2}$, one finds $(n+2)(2 n+1) / 2$ roots for even $n$ and $(n+1)(2 n+3) / 2$ roots for odd $n$. Among these roots, $n(n+3) / 2$ originate from the solutions of $U_{j}=0(j=1,2, \ldots, n)$ including one root associate with the mode described in Eq. (63), and the remains belong to the coupling modes. For quadrupole modes, there are $n / 2$ and $(n+1) / 2$ low-frequency coupling modes for even and odd $n$, respectively. When $k=0$, all the roots associated with the coupling modes degenerate into trivial solutions.

Here, we concentrate on the first 18 roots, i.e., the transverse modes up to $T_{2,3}$ found in using the $3 \times 3$ or higher truncations of the infinite determinants in the dispersion relation. The results presented in the following were actually computed by employing $4 \times 4$ determinants in Eq. (38). For zero wall impedance, $\left|\operatorname{Re}\left(\Omega / \nu_{o}\right)\right|$ is depicted in Figs. 7 and 8 as a function of $\nu / \nu_{o}$ for the

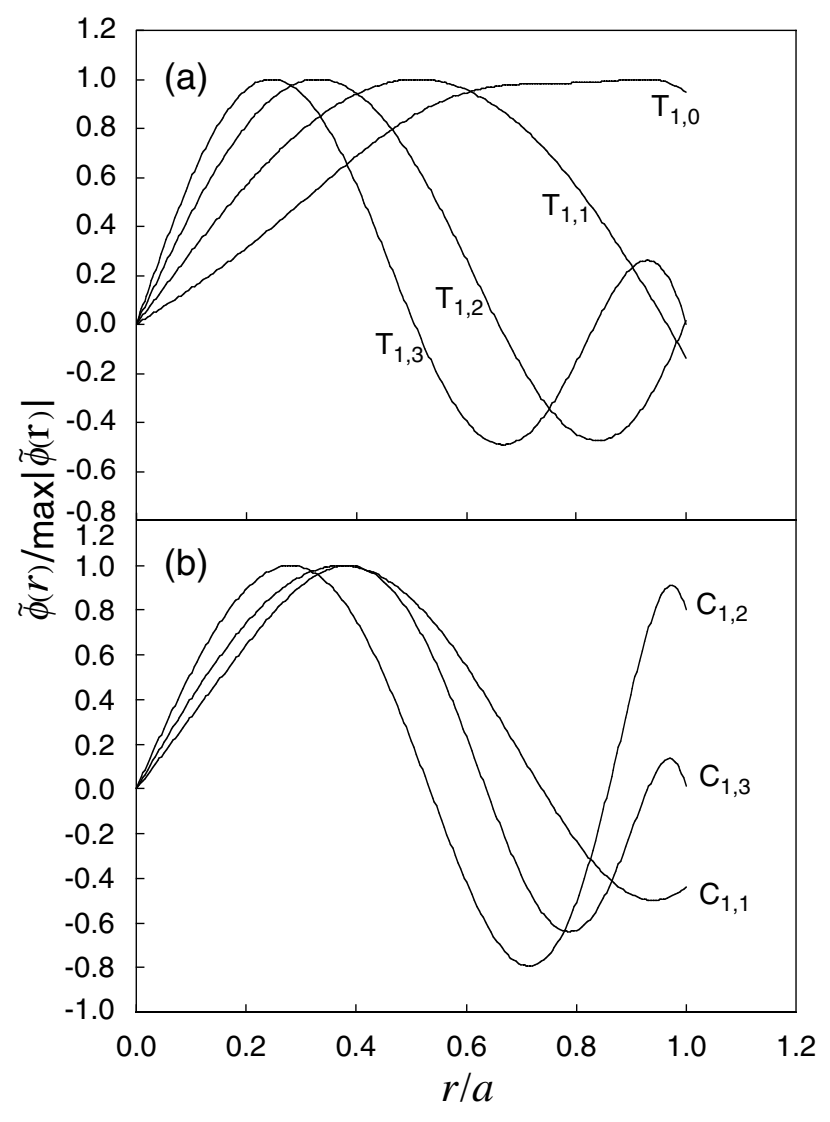

FIG. 6. Examples of the approximate radial mode structure as a function of $r / a$ for the two classes of dipole modes: (a) the usual dipole mode $T_{1,0}$, and the $T_{1,1}, T_{1,2}, T_{1,3}$ modes; (b) the high-frequency coupling modes. The case considered here is for $a k=2.0, b / a=1.5, \nu=0.697 \nu_{o}$, and zero wall impedance. The maximal amplitude is normalized to 1 .

cases of $k=0$ and $a k=2$, respectively. The corresponding $\left|\operatorname{Im}\left(\Omega / \nu_{o}\right)\right|$ is shown in Figs. 9 and 10. Also shown in Fig. 10 is the $\left|\operatorname{Im}\left(\Omega / \nu_{o}\right)\right|$ of the $T_{1,0}$ mode for the wall impedance of $Z=0.2$. Figure 11 displays the detail of the real part of $\Omega^{2} / \nu_{o}^{2}$ as a function of $\nu / \nu_{o}$ in the highintensity region for the case of $a k=2.0$. The approximate radial structures of the modes investigated are given in Fig. 12 where the results were computed by including the coefficients up to $A_{3}$ for $a k=2.0, \nu=0.6976 \nu_{o}$, and zero wall impedance.

As shown in Figs. 7 and 8, that when $\nu \rightarrow \nu_{o}$, the values of $\Omega / \nu_{o}$ approach zero and the solutions of $U_{j}=0(j=$ 1,2 , and 3), i.e., $0,2,4,6$, and 8. As $\nu$ increases, the values of the Doppler-shifted frequencies of the $T$ modes and the high-frequency coupling modes decrease. For $k>0$, the $\Omega$ of the low-frequency coupling modes starts from 0 at $\nu=\nu_{o}$ and increases to maxima in the region of $0.27<\nu / \nu_{o}<0.35$ before decreasing toward zero. Similar to the $m=1$ case, when $\nu \rightarrow 0$, the $T_{2,0}$ mode approaches the cold-beam limit, while the $\Omega / \nu_{o}$ of the highest $T_{2,3}, T_{2,2}$, and $T_{2,1}$ modes approaches 1.4, and the $\Omega$ of all other modes approaches zero. 


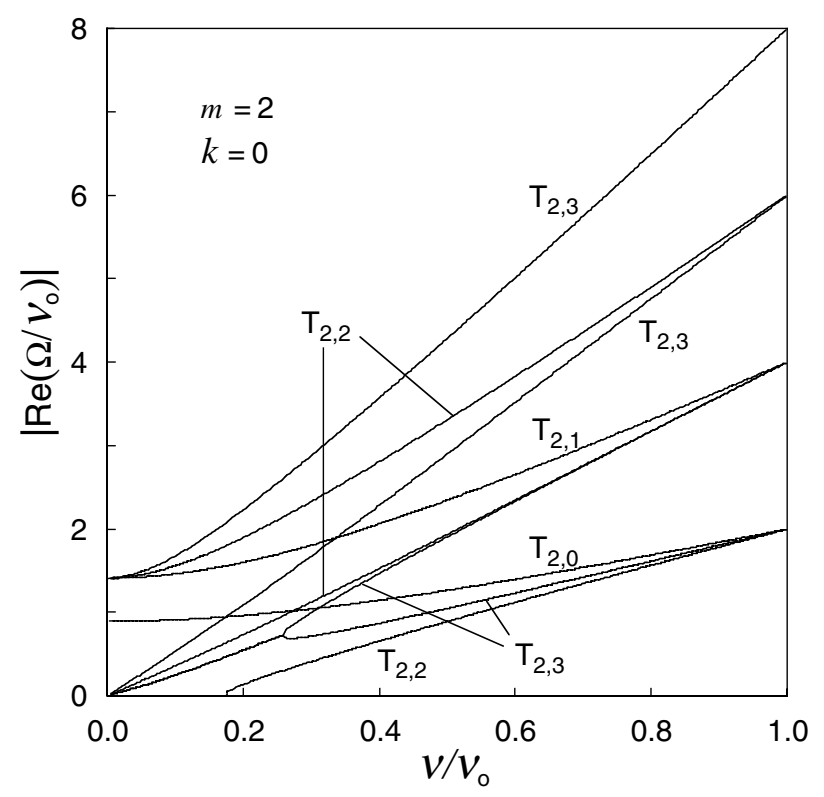

FIG. 7. Absolute value of the real part of $\Omega / \nu_{o}$ for the first nine quadrupole modes as a function of $\nu / \nu_{o}$ for $k=0, b / a=$ 1.5 , and zero wall impedance.

In the $k=0$ case, the $T_{2,2}$ mode becomes unstable when $\nu<0.177 \nu_{o}$ and we find a confluence of the two lower $T_{2,3}$ modes in the region of $0 \leq \nu<0.258 \nu_{o}$ with the highest growth rate around $0.15 \nu_{o}$. For $a k=2$, more

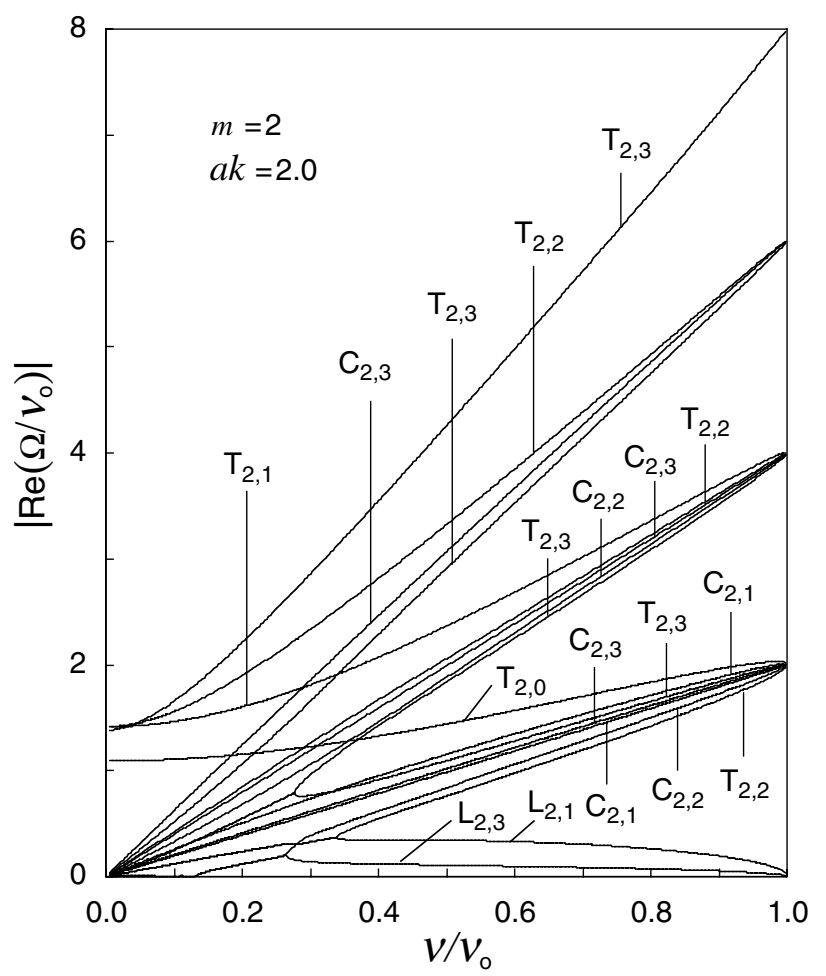

FIG. 8. Absolute value of the real part of $\Omega / \nu_{o}$ for the first 18 quadrupole modes as a function of $\nu / \nu_{o}$ for $a k=2.0, b / a=$ 1.5 , and zero wall impedance.

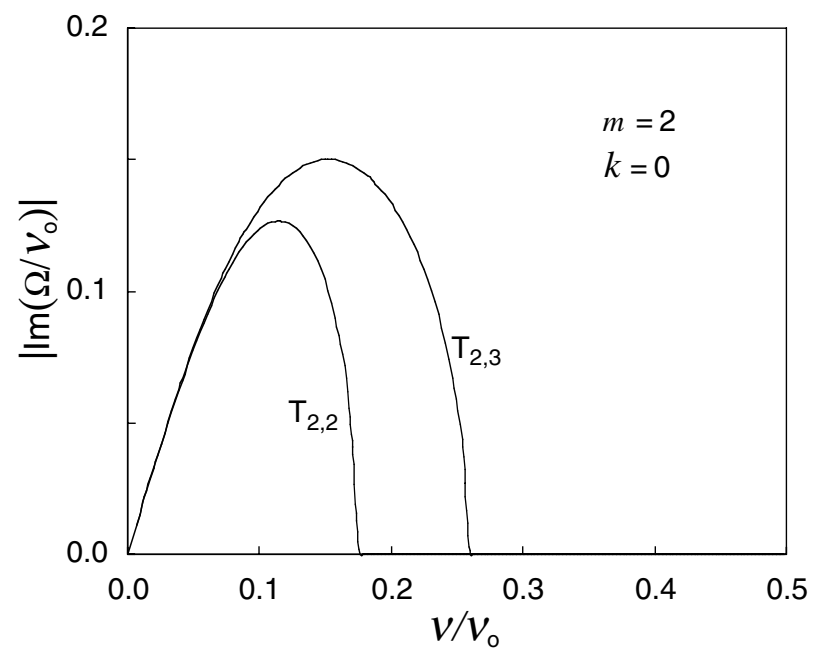

FIG. 9. Absolute value of the imaginary part of $\Omega / \nu_{o}$ for the first nine quadrupole modes as a function of $\nu / \nu_{o}$ for $k=0$ and $b / a=1.5$. Here, only the modes with none-zero $\operatorname{Im}\left(\Omega / \nu_{o}\right)$ are labeled.

confluences take place: the confluence of $T_{2,2}$ and $L_{2,1}$ $\left(0.28<\nu / \nu_{o}<0.34\right)$, the confluence of $C_{2,2}$ and $L_{2,1}$ $\left(0 \leq \nu<0.28 \nu_{o}\right)$, the confluence of $T_{2,2}$ and $L_{2,3}$ $\left(0.132<\nu / \nu_{o}<0.265\right)$, and the confluence of $T_{2,0}$ and $T_{2,3}$ near $\nu=0.357 \nu_{o}$. The real part of $\Omega$ in the confluence of $T_{2,2}$ and $L_{2,3}$ decreases to zero near $\nu=0.133 \nu_{o}$. These mode interactions are detailed in Figs. 10 and 11. We found that the confluence of the two lower $T_{2,3}$ has the highest growth rate $\left(\approx 0.153 \nu_{o}\right)$ among the $m=2$ modes studied. This highest growth rate, at the same order of magnitude of that of the $m=0$ modes [12], is considerably lower than the highest growth rate of the

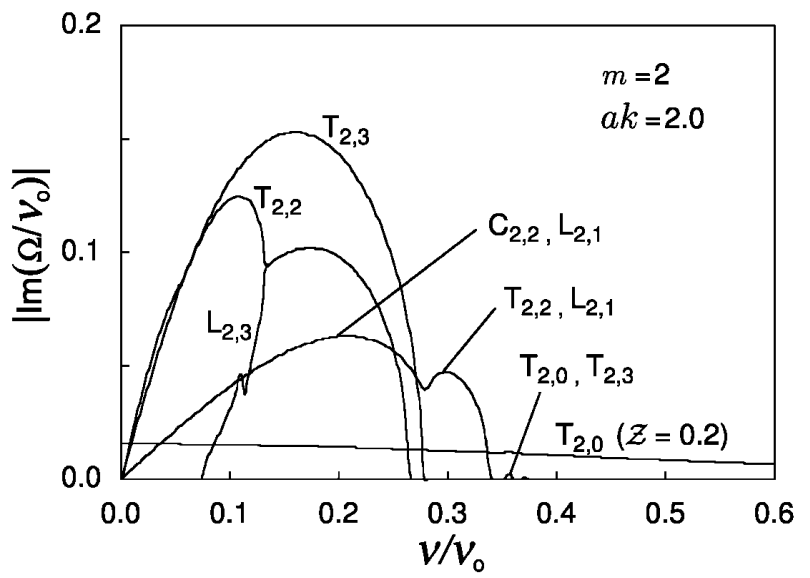

FIG. 10. Absolute value of the imaginary part of $\Omega / \nu_{o}$ for the first 18 quadrupole modes as a function of $\nu / \nu_{o}$ for $a k=$ $2.0, b / a=1.5$, and zero wall impedance. Only the modes with none-zero $\operatorname{Im}\left(\Omega / \nu_{o}\right)$ are labeled. Also shown is the absolute value of the imaginary part of $\Omega / \nu_{o}$ of the $T_{2,0}$ mode for the case of $Z=0.2$. The kink in the $L_{2,3}$ mode near $\nu=1.1 \nu_{o}$ is due to a confluence of $L_{2,3}$ and $T_{2,4}$ modes. 


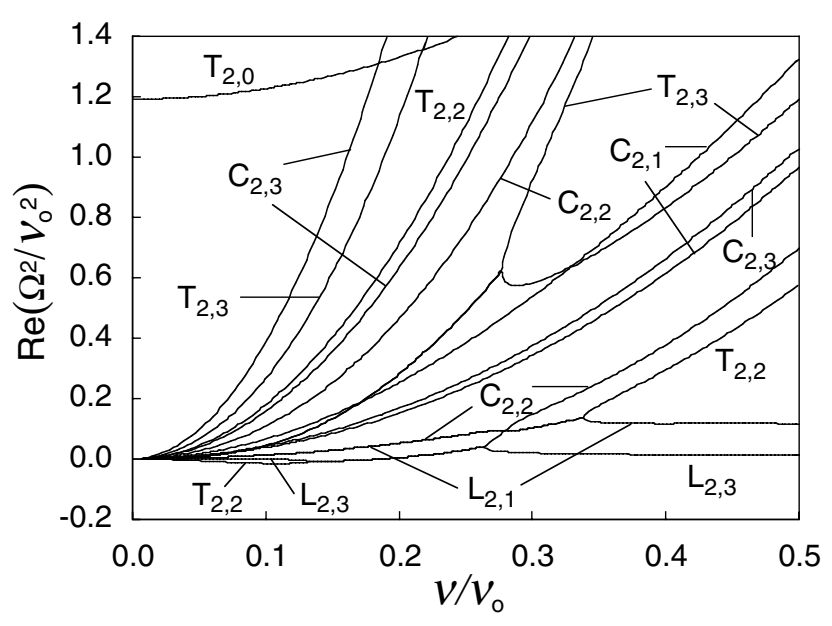

FIG. 11. The real part of $\Omega^{2} / \nu_{o}^{2}$ for the first 18 quadrupole modes as a function of $\nu / \nu_{o}$ for $a k=2.0, b / a=1.5$, and zero wall impedance.

$m=1$ modes. We also found that only the usual quadrupole mode, the $T_{2,0}$ mode, is appreciably influenced by the resistive wall impedance. The highest growth rate occurs near $\nu \approx 0$. For $Z=0.2$, as shown in Fig. 10 , the maximum growth rate of the $T_{2,0}$ mode is about $0.016 \nu_{o}$, approximately $70 \%$ of that of the usual dipole mode. A comparison between the $k=0$ and $a k=2$ cases reveals that the modes with more radial nodes are affected less by the longitudinal perturbation.

\section{CONCLUSIONS}

We have carried out a three-dimensional stability analysis for a continuous beam with axisymmetric KV distribution within the context of linearized VlasovMaxwell equations and electrostatic approximation. Although the model considered is simple, the coupled Vlasov-Poisson equations have been solved rigorously and the results should give a qualitative indication of the coupling between longitudinal and transverse perturbations. A dispersion relation has been derived to facilitate the investigation of the stability of any threedimensional mode. Several special cases and limits of the dispersion relation were discussed. Examples of numerical studies were presented for some lower radial modes of dipole $(m=1)$ and quadrupole $(m=2)$ perturbations.

It is found that in addition to the transverse modes discovered previously in the axially uniform perturbations, there also exist two classes of coupling modes due to the interaction between transverse and longitudinal motions. It was also found that the longitudinal perturbation affects the lower radial modes most. The dominant instabilities in the high-intensity region are originated from the pure transverse modes $T_{1,3}$ and $T_{2,2}$ as well as the confluence of $T_{1,3}$ modes and the confluence of $T_{2,3}$ modes. Our results show that the highest growth rate of the dipole modes is larger than that of the axisymmetric modes studied before. The interaction between

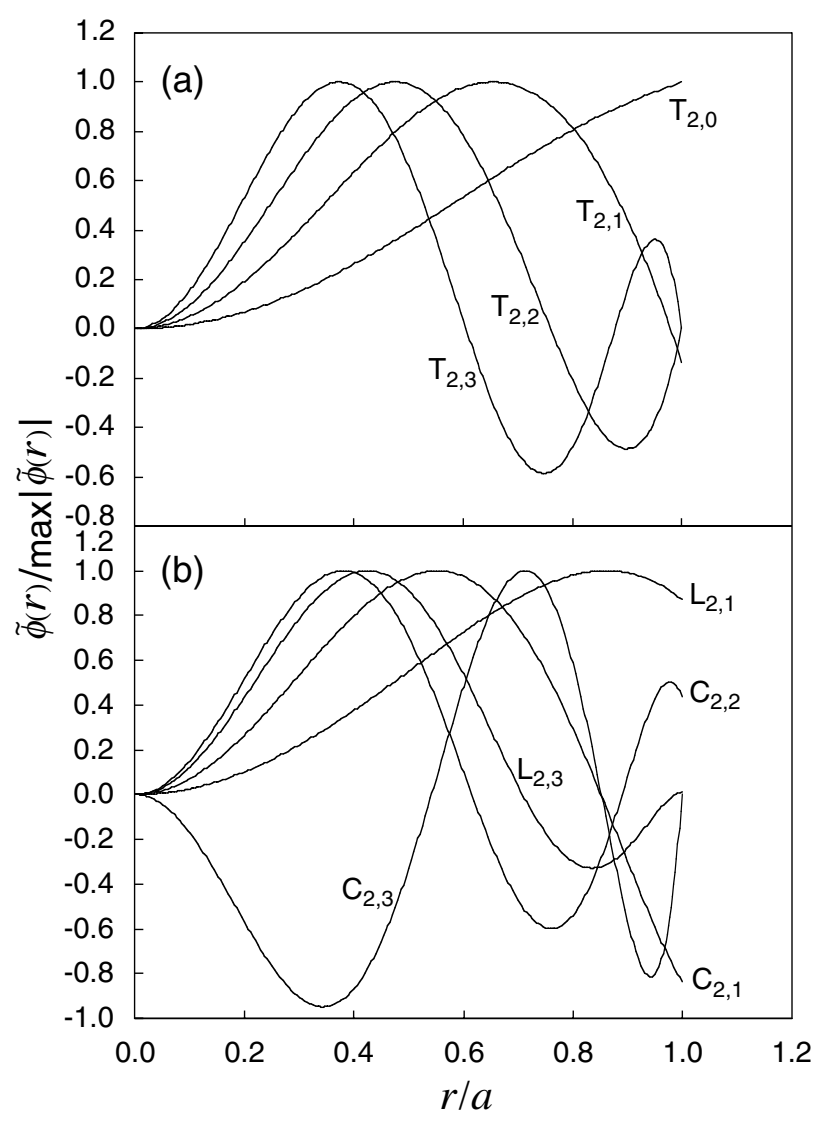

FIG. 12. Examples of the approximate radial mode structure as a function of $r / a$ for the three classes of quadrupole modes: (a) the usual quadrupole mode $T_{2,0}$, and the $T_{2,1}, T_{2,2}, T_{2,3}$ modes; (b) the high- and the low-frequency coupling modes. The case considered here is for $a k=2.0, b / a=1.5, \nu=$ $0.6976 \nu_{o}$, and zero wall impedance. The maximal amplitude is normalized to 1 .

longitudinal and transverse perturbations causes more mode confluences besides those mentioned. The frequencies in the confluent regions are complex conjugate pairs indicating possible instability. We discovered that some originally independent, stable transverse modes, e.g., the modes $T_{1,0}$ and $T_{1,2}$, can interact through longitudinal perturbation to cause instability. In the medium- and low-intensity regions, the interaction between longitudinal and transverse perturbations appears to be insignificant. Thus, except for the high-intensity regime, separate consideration of longitudinal and transverse modes tends to be a very good approximation. The effect of resistive wall impedance was also studied for dipole and quadrupole modes. We found that only the modes that involve surface motion, the usual dipole and quadrupole modes, are appreciably influenced by the resistive wall impedance.

Since not all instabilities predicted for a KV beam are realized in real beams, computer simulations are suggested for further investigation. In particular, the instability related to the confluences of modes of higher radial 
or azimuthal harmonics, e.g., the confluence of the two upper $T_{1,3}$ modes and the confluence of $T_{2,3}$ modes, perhaps can be found only in the KV distribution. This kind of instability or mode may not exist in beams with any phase space that differs from the KV distribution even at a small deviation. Since the growth rate of dipole modes is substantially higher than that of quadrupole modes, unstable dipole modes like $T_{1,0}$ or $T_{1,1}$, or the confluence of $T_{1,2}$ and $T_{1,0}$, have higher chances to be observed in simulations. The instability in the $T_{1,0}$ mode is driven by external impedance. It therefore can be seen at any beam intensity provided the impedance is sufficiently high. For zero external impedance, $T_{1,1}$ is more unstable than the confluence of $T_{1,2}$ and $T_{1,0}$. Simulations are more likely to find a growing $T_{1,1}$ mode. Take the result shown in Fig. 4 as an example, i.e., a perturbation of the $T_{1,1}$ mode structure with wavelength $\lambda=\pi a$. Instability with near zero or very-low Doppler-shifted frequency ( $\Omega \approx 0$ in the beam frame) may occur when the betatron frequency is depressed to below $0.55 \nu_{o}$. The unstable oscillation in the laboratory frame has a frequency near $2 v_{z} / a$. For perturbations of shorter wavelength, one can expect to find the instability at lower beam intensity with increased oscillation frequency and growth rate. Because of its relatively low growth rate, the instability due to the confluence of $T_{1,2}$ and $T_{1,0}$ modes may have to be seen in simulations with the mode structure seeded in the initial condition. For $\lambda=\pi a$, if this instability is observable, growing oscillations with $\Omega \approx$ $\nu_{o}$ should occur when the betatron frequency is depressed to near $0.46 \nu_{o}$.

As mentioned before, the model we have studied neglects the damping caused by energy spread that may substantially modify the growth rates reported here. Generalization of the present analysis to non-KV distribution and the effects of longitudinal velocity spread are still being pursued.

\section{ACKNOWLEDGMENTS}

This research was supported by the U.S. Department of Energy. The author thanks Dr. Andrew J. Jason for reading the manuscript and making suggestions for improvement.

\section{APPENDIX A}

In this appendix, we evaluate the Fourier component of the perturbed charge density shown in the right-hand side of Eq. (15) in the main text. Substituting Eq. (21) into the RHS of Eq. (15) yields

$$
\begin{aligned}
\mathrm{RHS}= & \frac{\omega_{p}^{2}}{a \nu^{2}} \delta(r-a) \sum_{l=0}^{\infty}(-1)^{l} G_{l} \\
& +\frac{2 \omega_{p}^{2}}{\pi} \sum_{l=0}^{\infty} G_{l} \int_{0}^{\infty} e^{-i \Omega \tau}\left(i \Omega J_{2}+\frac{k^{2} \tau}{2} J_{1}\right) d \tau
\end{aligned}
$$

where

$$
J_{1}=\int_{-\infty}^{\infty} \int_{-\infty}^{\infty} d v_{r} d v_{\varphi} \delta_{\perp}\left(\frac{\xi_{1}^{\prime}}{a} e^{i \theta}+\frac{\xi_{2}^{\prime}}{a}\right)^{m} P_{l}^{(m, 0)}\left(s^{\prime}\right)
$$

$$
J_{2}=\int_{-\infty}^{\infty} \int_{-\infty}^{\infty} d v_{r} d v_{\varphi}\left(\frac{d \delta_{\perp}}{d v_{\perp}^{2}}\right)\left(\frac{\xi_{1}^{\prime}}{a} e^{i \theta}+\frac{\xi_{2}^{\prime}}{a}\right)^{m} P_{l}^{(m, 0)}\left(s^{\prime}\right)
$$

$\delta_{\perp}=\delta\left[v_{\perp}^{2}-\nu^{2}\left(a^{2}-r^{2}\right)\right], \quad \xi_{1}^{\prime}$ and $\xi_{2}^{\prime}$ are given in Eqs. (8) and (9), respectively, $\theta=\cos ^{-1}\left(v_{r} / v_{\perp}\right), v_{\perp}=$ $\left(v_{r}^{2}+v_{\varphi}^{2}\right)^{1 / 2}, s^{\prime}=1-2\left(r^{\prime} / a\right)^{2}, r^{\prime}$ is given in Eq. (4), and the prime indicates the dependence on the unperturbed particle orbit.

We consider $J_{1}$ first by rewriting it as

$$
\begin{aligned}
J_{1} & =\frac{1}{2} \int_{0}^{2 \pi} d \theta \int_{-\infty}^{\infty} d v_{\perp}^{2} \delta\left[v_{\perp}^{2}-\nu^{2}\left(a^{2}-r^{2}\right)\right]\left(\frac{\xi_{1}^{\prime}}{a} e^{i \theta}+\frac{\xi_{2}^{\prime}}{a}\right)^{m} P_{l}^{(m, 0)}\left(1-\frac{2 r^{\prime 2}}{a^{2}}\right) \\
& =\frac{1}{2} \int_{0}^{2 \pi} d \theta\left[-\sqrt{1-(r / a)^{2}} \sin (\nu \tau) e^{i \theta}+(r / a) \cos (\nu \tau)\right]^{m} P_{l}^{(m, 0)}\left[s \cos (2 \nu \tau)+2(r / a) \sqrt{1-(r / a)^{2}} \sin (2 \nu \tau) \cos \theta\right]
\end{aligned}
$$

where $s=1-2(r / a)^{2}$. Letting $\psi=\theta+\pi, r / a=\sin \eta$, and $x=\nu \tau$, the integration in Eq. (A4) is then performed with the aid of Eq. (B1) in Appendix B to give

$$
\begin{aligned}
J_{1} & =\frac{1}{2} \int_{\pi}^{3 \pi} d \psi\left(\cos \eta \sin x e^{i \psi}+\sin \eta \cos x\right)^{m} P_{l}^{(m, 0)}[\cos (2 x) \cos (2 \eta)-\sin (2 x) \sin (2 \eta) \cos \psi] \\
& =\frac{1}{2} \int_{\pi}^{3 \pi} d \psi e^{i m \psi / 2} \sum_{\sigma=-j}^{j} e^{-i \sigma \psi} \sin ^{\alpha_{1}} \eta \cos ^{\beta_{1}} \eta \sin ^{\alpha_{2}} x \cos ^{\beta_{2}} x P_{j_{1}}^{\left(\alpha_{1}, \beta_{1}\right)}(\cos 2 \eta) P_{l}^{\left(\alpha_{2}, \beta_{2}\right)}(\cos 2 x) \\
& =\pi\left(\frac{r}{a}\right)^{m} P_{l}^{(m, 0)}(s) \cos ^{m}(\nu \tau) P_{l}^{(0, m)}(T),
\end{aligned}
$$

where $j_{1}=l+(m / 2)-\sigma, \alpha_{1}=\sigma+(m / 2), \beta_{1}=\sigma-(m / 2), j=l+(m / 2), \alpha_{2}=(m / 2)-\sigma, \beta_{2}=(m / 2)+\sigma$, and $T=\cos (2 \nu \tau)$ has been defined in Eq. (27) in the main text. 
Next, we consider $J_{2}$. Using integration by part, we can derive from Eq. (A3) that

where

$$
J_{2}=\frac{(-1)^{l+1} \pi}{2 a \nu^{2}} \delta(r-a) \cos ^{m}(\nu \tau) P_{l}^{(0, m)}(T)+\frac{1}{2}\left[\frac{m \sin x}{a \nu} J_{3}-\left(1-\delta_{l 0}\right) J_{4}\right],
$$

$$
J_{3}=\int_{0}^{2 \pi} d \theta \int_{0}^{\infty} d v_{\perp} \delta_{\perp} e^{i \theta}\left(\frac{\xi_{1}^{\prime}}{a} e^{i \theta}+\frac{\xi_{2}^{\prime}}{a}\right)^{m-1} P_{l}^{(m, 0)}\left(s^{\prime}\right),
$$

and

$$
J_{4}=\int_{0}^{2 \pi} d \theta \int_{0}^{\infty} d v_{\perp} \delta_{\perp}\left(\frac{\xi_{1}^{\prime}}{a} e^{i \theta}+\frac{\xi_{2}^{\prime}}{a}\right)^{m}\left(\frac{\partial s^{\prime}}{\partial v_{\perp}}\right) \frac{\partial P_{l}^{(m, 0)}\left(s^{\prime}\right)}{\partial s^{\prime}} .
$$

Applying Eqs. (B1) and (C14) to Eq. (A7), we have

$$
\begin{aligned}
J_{3} & =\sum_{j=0}^{l} \frac{(m+2 j) l !(m+j-1) !}{j !(m+l) !} \int_{0}^{2 \pi} d \theta \int_{0}^{\infty} d v_{\perp} \delta_{\perp} e^{i \theta}\left(\frac{\xi_{1}^{\prime}}{a} e^{i \theta}+\frac{\xi_{2}^{\prime}}{a}\right)^{m-1} P_{j}^{(m-1,0)}\left(s^{\prime}\right) \\
& =\frac{-1}{2 \nu \sqrt{a^{2}-r^{2}}} \sum_{j=0}^{l} \frac{(m+2 j) l !(m+j-1) !}{j !(m+l) !} \int_{\pi}^{3 \pi} d \psi e^{i \psi(m+1) / 2} \sum_{\sigma=-n}^{n} e^{-i \sigma \psi} \sin ^{\alpha_{1}} \eta \cos ^{\beta_{1}} \eta \sin ^{\alpha_{2}} x \cos ^{\beta_{2}} x P_{n_{1}}^{\left(\alpha_{1}, \beta_{1}\right)}(\cos 2 \eta) P_{j}^{\left(\alpha_{2}, \beta_{2}\right)}(T) \\
& =\frac{-\pi}{a \nu \sin x}\left(\frac{r}{a}\right)^{m} \cos ^{m} x \sum_{j=1}^{l} \frac{l !(m+j-1) !}{j !(m+l) !}(m+2 j) P_{j-1}^{(m, 1)}(s) P_{j}^{(-1, m)}(T),
\end{aligned}
$$

where $\psi=\theta+\pi, j$ and $m$ are not simultaneously equal to zero, $n_{1}=j-\sigma+[(m-1) / 2], \alpha_{1}=\sigma+[(m-1) / 2]$, $\beta_{1}=\sigma-[(m-1) / 2], n=j+[(m-1) / 2], \alpha_{2}=[(m-1) / 2]-\sigma$, and $\beta_{2}=[(m-1) / 2]+\sigma$. The integral $J_{4}$ can be treated by using Eq. (C15) to yield

$$
J_{4}=\frac{2 J_{5}}{a^{2} \nu}\left[\frac{l ! m !}{(m+l) !}+(-1)^{l+1}\right](m+1) \sin x+\frac{2 \sin x}{a^{2} \nu^{2}} \sum_{j=1}^{l-1}\left[\frac{l !(m+j) !}{j !(m+l) !}+(-1)^{l+j+1}\right](m+2 j+1)\left(\nu J_{6} \cos x-J_{1} \sin x\right),
$$

where $J_{1}$ is given in Eq. (A5),

$$
\begin{aligned}
J_{5} & =r \cos x \int_{0}^{2 \pi} d \theta \int_{0}^{\infty} d v_{\perp} \delta_{\perp} \cos \theta\left(\frac{\xi_{1}^{\prime}}{a} e^{i \theta}+\frac{\xi_{2}^{\prime}}{a}\right)^{m} P_{0}^{(m, 0)}\left(s^{\prime}\right) \\
& =\frac{m \pi r \cos x}{a} \int_{0}^{\infty} \delta\left[v_{\perp}^{2}-\nu^{2}\left(a^{2}-r^{2}\right)\right]\left(\frac{\xi_{2}^{\prime}}{a}\right)^{m-1} \xi_{1}^{\prime} d v_{\perp}=\frac{-m \pi \sin x}{2 \nu}\left(\frac{r}{a}\right)^{m} \cos ^{m} x
\end{aligned}
$$

and

$$
\begin{aligned}
J_{6} & =r \int_{0}^{2 \pi} d \theta \int_{0}^{\infty} d v_{\perp} \delta_{\perp} \cos \theta\left(\frac{\xi_{1}^{\prime}}{a} e^{i \theta}+\frac{\xi_{2}^{\prime}}{a}\right)^{m} P_{j}^{(m, 0)}\left(s^{\prime}\right) \\
& =\frac{-\pi r}{2 \nu a \sin x}\left(\frac{r}{a}\right)^{m+1} \cos ^{m+1} x P_{j-1}^{(m+1,1)}(s) P_{j}^{(-1, m+1)}(T)-\frac{\pi a^{2}}{2 \nu\left(a^{2}-r^{2}\right)}\left(\frac{r}{a}\right)^{m} \cos ^{m} x \sin x P_{j+1}^{(m-1,-1)}(s) P_{j}^{(1, m-1)}(T) .
\end{aligned}
$$

Applying the results in Eqs. (A9)-(A12) to Eq. (A6) and then using Eqs. (C1), (C2), (C4), (C5), and (C17), we evaluate the integral $J_{2}$ as

$$
\begin{aligned}
J_{2}= & \frac{(-1)^{l+1} \pi}{2 a \nu^{2}} \delta(r-a) \cos ^{m}(\nu \tau) P_{l}^{(0, m)}(T) \\
& -\frac{\pi\left(1-\delta_{l 0}\right)}{a^{2} \nu^{2}}\left(\frac{r}{a}\right)^{m} \cos ^{m}(\nu \tau) \sum_{j=1}^{l-1}(-1)^{l+j}(m+j+1)\left[P_{j}^{(0, m)}(T)-P_{j+1}^{(0, m)}(T)\right] P_{j}^{(m, 1)}(s) .
\end{aligned}
$$

Substituting Eqs. (A5) and (A13) into Eq. (A1) leads to Eq. (26) in the main text. 


\section{APPENDIX B}

Here, we prove the relation: if $\cos \theta=\cos \theta_{1} \cos \theta_{2}-\sin \theta_{1} \sin \theta_{2} \cos \phi$, for $0 \leq \theta \leq \pi, 0 \leq \theta_{1} \leq \pi, 0 \leq \theta_{2} \leq \pi$, and $0 \leq \phi \leq 2 \pi$, then

$$
\begin{aligned}
& {\left[\cos \left(\theta_{1} / 2\right) \cos \left(\theta_{2} / 2\right) e^{-i \phi / 2}-\sin \left(\theta_{1} / 2\right) \sin \left(\theta_{2} / 2\right) e^{i \phi / 2}\right]^{\beta}} \\
& \quad \times\left[\sin \left(\theta_{1} / 2\right) \cos \left(\theta_{2} / 2\right) e^{-i \phi / 2}+\cos \left(\theta_{1} / 2\right) \sin \left(\theta_{2} / 2\right) e^{i \phi / 2}\right]^{\alpha} P_{k}^{(\alpha, \beta)}(\cos \theta) \\
& =\sum_{\sigma=-l}^{l} e^{-i \sigma \phi} \sin ^{\alpha_{1}}\left(\theta_{1} / 2\right) \cos ^{\beta_{1}}\left(\theta_{1} / 2\right) \sin ^{\alpha_{2}}\left(\theta_{2} / 2\right) \cos ^{\beta_{2}}\left(\theta_{2} / 2\right) P_{k_{1}}^{\left(\alpha_{1}, \beta_{1}\right)}\left(\cos \theta_{1}\right) P_{k}^{\left(\alpha_{2}, \beta_{2}\right)}\left(\cos \theta_{2}\right)
\end{aligned}
$$

where $k_{1}=k-\sigma+(\alpha+\beta) / 2, l=k+(\alpha+\beta) / 2, \alpha_{1}=\sigma-(\beta-\alpha) / 2, \alpha_{2}=-\sigma+(\alpha+\beta) / 2, \beta_{1}=\sigma+(\beta-$ $\alpha) / 2$, and $\beta_{2}=\sigma+(\alpha+\beta) / 2$.

The proof in the following invokes an addition relation of the generalized spherical function developed in the group representation theory [42]. The generalized spherical function defined by

$$
P_{m n}^{l}(z)=\frac{i^{n-m}(-1)^{l-n}}{2^{l}}\left[\frac{(l+m) !}{(l-n) !(l+n) !(l-m) !}\right]^{1 / 2}(1+z)^{-(m+n) / 2}(1-z)^{-(m-n) / 2} \frac{d^{l-m}}{d z^{l-m}}\left[(1-z)^{l-n}(1+z)^{l+n}\right],
$$

has the symmetry property $P_{m n}^{l}(z)=P_{n m}^{l}(z)$, and is related to Jacobi polynomials by

$$
P_{m n}^{l}(z)=2^{-n} i^{\alpha}\left[\frac{(l-n) !(l+n) !}{(l-m) !(l+m) !}\right]^{1 / 2}(1-z)^{\alpha / 2}(1+z)^{\beta / 2} P_{l-n}^{(\alpha, \beta)}(z),
$$

where $\alpha=n-m$ and $\beta=n+m$. The addition relation of the generalized spherical functions [42], quoted here without proof, states that if

$$
\begin{gathered}
\cos \theta=\cos \theta_{1} \cos \theta_{2}-\sin \theta_{1} \sin \theta_{2} \cos \phi, \\
e^{i \phi^{\prime}}=\left[\sin \theta_{1} \cos \theta_{2}+\cos \theta_{1} \sin \theta_{2} \cos \phi+i \sin \theta_{2} \sin \phi\right] / \sin \theta,
\end{gathered}
$$

and

$$
\cos (\theta / 2) e^{i\left(\phi^{\prime}+\psi\right) / 2}=\cos \left(\theta_{1} / 2\right) \cos \left(\theta_{2} / 2\right) e^{i \phi / 2}-\sin \left(\theta_{1} / 2\right) \sin \left(\theta_{2} / 2\right) e^{-i \phi / 2},
$$

for $0 \leq \theta, \theta_{1}, \theta_{2}, \phi, \phi^{\prime} \leq 2 \pi$, and $-2 \pi \leq \psi \leq 2 \pi$, then

$$
e^{-i\left(m \phi^{\prime}+n \psi\right)} P_{m n}^{l}(\cos \theta)=\sum_{s=-l}^{l} e^{-i s \phi} P_{m s}^{l}\left(\cos \theta_{1}\right) P_{s n}^{l}\left(\cos \theta_{2}\right) .
$$

To prove Eq. (B1), we notice that if relation (B4) is true, then

$$
\cos (\theta / 2)=\Lambda^{1 / 2}\left[\cos \left(\theta_{1} / 2\right) \cos \left(\theta_{2} / 2\right) e^{-i \phi / 2}-\sin \left(\theta_{1} / 2\right) \sin \left(\theta_{2} / 2\right) e^{i \phi / 2}\right]^{1 / 2},
$$

and

$$
\sin (\theta / 2)=\Xi^{1 / 2}\left[\sin \left(\theta_{1} / 2\right) \cos \left(\theta_{2} / 2\right) e^{i \phi / 2}+\cos \left(\theta_{1} / 2\right) \sin \left(\theta_{2} / 2\right) e^{-i \phi / 2}\right]^{1 / 2},
$$

where

$$
\Lambda=\cos \left(\theta_{1} / 2\right) \cos \left(\theta_{2} / 2\right) e^{i \phi / 2}-\sin \left(\theta_{1} / 2\right) \sin \left(\theta_{2} / 2\right) e^{-i \phi / 2}
$$

and

$$
\Xi=\cos \left(\theta_{1} / 2\right) \sin \left(\theta_{2} / 2\right) e^{i \phi / 2}+\sin \left(\theta_{1} / 2\right) \cos \left(\theta_{2} / 2\right) e^{-i \phi / 2} .
$$

One can also show that if relations (B5) and (B6) are true, then

$$
\sin (\theta / 2) e^{i\left(\phi^{\prime}-\psi\right) / 2}=\Xi \text {. }
$$

From Eqs. (B6) and (B8)-(B12) we derive

$$
\begin{aligned}
& 1+w=2 \Lambda\left[\cos \left(\theta_{1} / 2\right) \cos \left(\theta_{2} / 2\right) e^{-i \phi / 2}-\sin \left(\theta_{1} / 2\right) \sin \left(\theta_{2} / 2\right) e^{i \phi / 2}\right], \\
& 1-w=2 \Xi\left[\sin \left(\theta_{1} / 2\right) \cos \left(\theta_{2} / 2\right) e^{i \phi / 2}+\cos \left(\theta_{1} / 2\right) \sin \left(\theta_{2} / 2\right) e^{-i \phi / 2}\right],
\end{aligned}
$$


and

$$
e^{-i\left(m \phi^{\prime}+n \psi\right)}=\left(\frac{1+w}{2 \Lambda^{2}}\right)^{(m+n) / 2}\left(\frac{1-w}{2 \Xi^{2}}\right)^{(m-n) / 2},
$$

where $w=\cos \theta$. Letting $x=\cos \theta_{1}, y=\cos \theta_{2}$, and using Eqs. (B3)-(B6), we can rewrite Eq. (B7) as

$$
\begin{aligned}
e^{-i\left(m \phi^{\prime}+n \psi\right)}(1-w)^{(n-m) / 2}(1+w)^{(m+n) / 2} P_{k}^{(\alpha, \beta)}(w)= & \sum_{s=-l}^{l} e^{-i s \phi} 2^{-s}(1-x)^{(s-m) / 2}(1+x)^{(s+m) / 2}(1-y)^{(n-s) / 2} \\
& \times(1+y)^{(n+s) / 2} P_{k_{1}}^{\left(\alpha_{1}, \beta_{1}\right)}(x) P_{k_{2}}^{\left(\alpha_{2}, \beta_{2}\right)}(y),
\end{aligned}
$$

where $k_{1}=l-s=k-s+(\alpha+\beta) / 2, k_{2}=k, \alpha_{1}=s-m=s-(\beta-\alpha) / 2, \alpha_{2}=n-s=-s+(\alpha+\beta) / 2, \beta_{1}=$ $s+m=s+(\beta-\alpha) / 2, \beta_{2}=n+s=s+(\alpha+\beta) / 2, k=l-n, \alpha=n-m$, and $\beta=n+m$. Applying Eqs. (B13) (B15) to the left-hand side of Eq. (B16), and then making the substitutions of $1-x=2 \sin ^{2}\left(\theta_{1} / 2\right), 1+x=2 \cos ^{2}\left(\theta_{1} / 2\right)$, $1-y=2 \sin ^{2}\left(\theta_{2} / 2\right)$, and $1+y=2 \cos ^{2}\left(\theta_{2} / 2\right)$ on the right-hand side of the resulted equation leads to Eq. (B1).

\section{APPENDIX C}

In this appendix, we list the identities of Jacobi polynomials used in the main text and in the appendices. The following basic relations among Jacobi polynomials are included here without proof $[43,44]$ :

$$
\begin{gathered}
(\alpha+\beta+2 n) P_{n}^{(\alpha, \beta-1)}(x)=(\alpha+\beta+n) P_{n}^{(\alpha, \beta)}(x)+(\alpha+n) P_{n-1}^{(\alpha, \beta)}(x), \\
(\alpha+\beta+2 n) P_{n}^{(\alpha-1, \beta)}(x)=(\alpha+\beta+n) P_{n}^{(\alpha, \beta)}(x)-(\beta+n) P_{n-1}^{(\alpha, \beta)}(x), \\
\frac{1}{2}(2+\alpha+\beta+2 n)(1+x) P_{n}^{(\alpha, \beta+1)}(x)=(n+1) P_{n+1}^{(\alpha, \beta)}(x)+(n+1+\beta) P_{n}^{(\alpha, \beta)}(x), \\
\left(\frac{1-x}{2}\right) P_{l-1}^{(m+1,1)}(x)=\frac{1}{m+2 l+1}\left[(m+l) P_{l-1}^{(m, 1)}(x)-l P_{l}^{(m, 1)}(x)\right], \\
\left(\frac{1-x}{2}\right) P_{l}^{(1, m)}(x)=\frac{l}{m+2 l}\left[P_{l-1}^{(0, m)}(x)-P_{l}^{(0, m)}(x)\right], \\
\frac{d}{d y} P_{n}^{(\alpha, \beta)}(y)=\frac{1}{2}(\alpha+\beta+n+1) P_{n-1}^{(\alpha+1, \beta+1)}(y)=\frac{1}{2}\left[(n+\beta) P_{n-1}^{(\alpha+1, \beta)}(y)+(n+\alpha) P_{n-1}^{(\alpha, \beta+1)}(y)\right], \\
(y-1) \frac{d}{d y} P_{l}^{(m, 0)}(y)=l P_{l}^{(m, 0)}(y)-(m+l) P_{l-1}^{(m, 1)}(y), \\
\int_{-1}^{1}(1-x)^{\alpha}(1+x)^{\beta} P_{n}^{(\alpha, \beta)}(x) P_{m}^{(\alpha, \beta)}(x) d x=\left\{\begin{array}{l}
0, \\
\frac{2^{1+\alpha+\beta} \Gamma(1+\alpha+n) \Gamma(1+\beta+n)}{n !(1+\alpha+\beta+2 n) \Gamma(1+\alpha+\beta+n)},
\end{array}\right. \\
P_{n}^{(\alpha, \beta)}(-x)=(-1)^{n} P_{n}^{(\beta, \alpha)}(x), \\
P_{n}^{(\alpha, \beta)}(1)=(1+\alpha)_{n} / n !, \\
\text { for } n=m,
\end{gathered}
$$

and

$$
P_{j}^{(0, m)}(\cos 2 x)-P_{j-1}^{(0, m)}(\cos 2 x)=\frac{(-1)^{j}(m+2 j)(j+m-1) !}{m ! j !}{ }_{2} F_{1}\left(-j, m+j ; m+1 ; \cos ^{2} x\right),
$$

where $\Gamma(x)$ is the Gamma function, $(x)_{j}=\Gamma(x+j) / \Gamma(x)$, and ${ }_{2} F_{1}(a, b ; c ; z)$ is the hypergeometric function. 
The next seven identities, used in this work, will be proved in this appendix:

$$
\begin{gathered}
P_{l}^{(m, 1)}(x)=\sum_{j=0}^{l} \frac{(-1)^{l-j}(m+2 j+1)}{m+l+1} P_{j}^{(m, 0)}(x), \\
P_{l}^{(m, 2)}(x)=\sum_{j=0}^{l-1} \frac{(-1)^{l-j-1}(m+2 j+2)(m+j+1)}{(m+l+1)(m+l)} P_{j}^{(m, 1)}(x), \\
P_{l}^{(m+1,0)}(x)=\sum_{j=0}^{l} \frac{l !(m+j) !}{j !(m+l+1) !}(m+2 j+1) P_{j}^{(m, 0)}(x), \\
\frac{d}{d x} P_{n}^{(m, 0)}(x)=\frac{1}{2} \sum_{j=0}^{n-1}\left[\frac{n !(m+j) !}{j !(m+n) !}-(-1)^{n+j}\right](m+2 j+1) P_{j}^{(m, 0)}(x), \\
\frac{d}{d y}\left[m P_{l}^{(m, 0)}(y)+(y-1) \frac{d P_{l}^{(m, 0)}(y)}{d y}\right]=\frac{1}{2} \sum_{j=0}^{l-1}(-1)^{l+j+1}(2 j+m+2)(j+m+1) P_{j}^{(m, 1)}(y), \\
P_{l+1}^{(m-1,-1)}(x)=\frac{(m+l)(1+x)}{2(l+1)(m+2 l+1)}\left[(m+l+1) P_{l}^{(m, 1)}(x)-(l+l) P_{l-1}^{(m, 1)}(x)\right],
\end{gathered}
$$

and

$$
I_{m}(k r)=\frac{1}{2}\left(\frac{r}{a}\right)^{m} \sum_{l=0}^{\infty}(-1)^{l}\left[I_{2 l+m}(a k)-I_{2 l+m+2}(a k)\right] P_{l}^{(m, 0)}\left(1-\frac{2 r^{2}}{a^{2}}\right),
$$

for $r \leq a$, where $I_{n}(x)$ is the modified Bessel function of the first kind.

To proof Eq. (C12), we apply Eq. (C1) iteratively to obtain

$$
\begin{aligned}
P_{n}^{(m, 1)}(x)= & \frac{1}{m+n+1}\left[(m+2 n+1) P_{n}^{(m, 0)}(x)-(m+n) P_{n-1}^{(m, 1)}(x)\right] \\
= & \left(\frac{m+2 n+1}{m+n+1}\right) P_{n}^{(m, 0)}(x)-\frac{1}{m+n+1}\left[(m+1+2 n-2) P_{n-1}^{(m, 0)}(x)-(m+n-1) P_{n-2}^{(m, 1)}(x)\right] \\
= & \sum_{k=n-1}^{n} \frac{(-1)^{n-k}(2 k+m+1)}{m+n+1} P_{k}^{(m, 0)}(x)+\left(\frac{m+n-1}{m+n+1}\right) P_{n-2}^{(m, 1)}(x) \\
& \cdots \cdots \cdots \cdots \cdots \cdots \cdots \cdots \cdots \cdots \cdots \cdots \cdots \cdots \cdots \cdots \cdots \cdots \cdots \\
= & \sum_{k=0}^{n} \frac{(-1)^{n-k}(2 k+m+1)}{m+n+1} P_{k}^{(m, 0)}(x) .
\end{aligned}
$$

Thus Eq. (C12) is proved. Equation (C19) shows a procedure of expanding $P_{n}^{(m, 1)}(x)$ in terms of $P_{k}^{(m, 0)}(x)$. Using the same procedure, we can expand $P^{(m, 2)}(x)$ in terms of $P_{k}^{(m, 1)}(x)$ to derive Eq. (C13). Similarly, one can prove Eq. (C14) by applying Eq. (C2) to $P_{n}^{(m+1,0)}(x)$ and the subsequent results repeatedly. Equation (C15) can be verified simply by substituting $\alpha=m, \beta=0$, Eqs. (C12) and (C14) into Eq. (C6).

The proof of Eq. (C16) is proceeded by applying Eq. (C7) to derive

$$
\frac{d}{d y}\left[m P_{l}^{(m, 0)}(y)+(y-1) \frac{d P_{l}^{(m, 0)}(y)}{d y}\right]=(m+l) \frac{d}{d y}\left[P_{l}^{(m, 0)}(y)-P_{l-1}^{(m, 1)}(y)\right]=(m+l) \frac{d}{d y} P_{l}^{(m-1,1)},
$$

where we have also used Eqs. (C1) and (C2). Equation (C16) is then obtained by applying Eqs. (C6) and (C13) to Eq. (C20).

To prove Eq. (C17), we first apply Eq. (C3) to $P_{j+1}^{(m-1,-1)}(x)$ and the subsequent results repeatedly to yield

$$
P_{j+1}^{(m-1,-1)}(x)=\left(\frac{x+1}{2}\right) \frac{(-1)^{j}}{(j+1)} \sum_{n=0}^{j}(-1)^{n}(m+2 n) P_{n}^{(m-1,0)}(x) .
$$

Next, using Eq. (C2) we can derive from Eq. (C21) that 


$$
P_{j+1}^{(m-1,-1)}(x)=\frac{x+1}{2}\left[\left(\frac{m+j}{j+1}\right) P_{j}^{(m, 0)}(x)+\sum_{n=0}^{j-1} \frac{(-1)^{j+n}(2 n+m+1)}{(j+1)} P_{n}^{(m, 0)}(x)\right]
$$

Applying Eq. (C1) to the right-hand side of Eq. (C22), we obtain Eq. (C17).

To verify Eq. (C18), we use the following integral involves Legendre polynomial $P_{l}(x)$ and Bessel function $J_{0}(x)[45]:$

$$
\int_{0}^{\pi / 2} \sin (2 \theta) P_{l}(\cos 2 \theta) J_{0}(\alpha \sin \theta) d \theta=\alpha^{-1} J_{2 l+1}(\alpha)
$$

together with the relation $J_{n}(i x)=i^{n} I_{n}(x)$ to expand $I_{0}(k r)$ in terms of Jacobi polynomials as

$$
I_{0}(k r)=\frac{1}{2} \sum_{l=0}^{\infty}(-1)^{l}\left[I_{2 l}(a k)-I_{2 l+2}(a k)\right] P_{l}^{(0,0)}\left(1-\frac{2 r^{2}}{a^{2}}\right),
$$

where $r \leq a$. Taking the derivative of Eq. (C24) with respect to $r$, we derive

$I_{1}(k r)=\frac{r}{2 a} \sum_{l=0}^{\infty}(-1)^{l}\left[I_{2 l+1}(a k)-I_{2 l+3}(a k)\right] P_{l}^{(1,0)}\left(1-\frac{2 r^{2}}{a^{2}}\right)$,

for $r \leq a$. Equation (C18) can then be derived from Eqs. (C24) and (C25) by using a mathematical induction method and taking the derivatives of Bessel functions.

[1] See, for example, Proceedings of the 2001 Particle Accelerator Conference, New York (IEEE, Piscataway, NJ, 2001), pp. 1-4097.

[2] See, for example, Proceedings of the 14th International Heavy Ion Fusion Symposium [Laser Part. Beams, 20, 367 (2002)], and references therein.

[3] R. A. Kishek, S. Bernal, C. L. Bohn, D. Grot, I. Haber, P. G. O'Shea, M. Reiser, and M. Walter, Phys. Plasmas 10, 2016 (2003).

[4] J. D. Lawson, The Physics of Charged-Particle Beams (Clarendon Press, Oxford, 1988).

[5] A.W. Chao, Physics of Collective Beam Instabilities in High Energy Accelerators (John Wiley \& Son, Inc., New York, 1992).

[6] S. Y. Lee, Accelerator Physics (World Science Publishing Co. Pte. Ltd., River Edge, NJ, 1999).

[7] R. C. Davidson and H. Qin, Physics of Intense Charged Particle Beams in High Energy Accelerators (Imperial College Press and World Scientific Publishing Co., London, 2001).

[8] R. L. Gluckstern, in Proceedings of the 1970 Proton Linear Accelerator Conference, IL, 1971, edited by M. R. Tracy (National Accelerator Laboratory, Batavia, 1971), p. 811.
[9] A.H.W. Beck, Space-Charge Waves and Slow Electromagnetic Waves (Pergamon Press, New York, 1958).

[10] M. J. Lee, F. E. Mills, and P. L. Morton, SLAC Report No. SLAC-76, 1967.

[11] G. Krafft, J.W.-K. Mark, L. Smith, and T. F. Wang, SIAM J. Appl. Math. 43, 1390 (1983).

[12] T. F. Wang and L. Smith, Part. Accel. 12, 247 (1982).

[13] I. M. Kapchinskij and V.V. Vladimirskij, in Proceedings of the 2nd International Conference on High Energy Accelerators and Instrumentation, 1959 (CERN Scientific Information Service, Geneva, 1959), p. 274.

[14] I. Haber, D. A. Callahan, A. Friedman, D. P. Grote, and A. B. Langdon, Fusion Eng. Des. 32-33, 169 (1996).

[15] I. Haber, D. A. Callahan, A. Friedman, D. P. Grote, S. M. Lund, and T. F. Wang, Nucl. Instrum. Methods Phys. Res., Sect. A 415, 405 (1998).

[16] S. M. Lund, D. A. Callahan, A. Friedman, D. P. Grote, I. Haber, and T.F. Wang, in Proceedings of the XIX International Linear Accelerator Conference, Chicago, IL, 1998, edited by C. E. Eyberger, R. C. Pardo, and M. M. White (Argonne National Laboratory, Argonne, IL, 1998), p. 372.

[17] E. G. Harris, Phys. Rev. Lett., 2, 34 (1959).

[18] S. M. Lund and R. C. Davidson, Phys. Plasmas 5, 3028 (1998).

[19] I. Haber, A. Friedman, D. P. Grote, S. M. Lund, and R. A. Kishek, Phys. Plasmas 6, 2254 (1999).

[20] H. Qin, R. C. Davidson, and W.W.-L. Lee, Phys. Rev. ST Accel. Beams 3, 084401 (2000).

[21] R. C. Davidson and S. Strasburg, Phys. Plasmas 7, 2657 (2000).

[22] R. A. Kishek, S. Bernal, P. G. O'Shea, M. Reiser, and I. Haber, Nucl. Instrum. Methods Phys. Res., Sect. A 464, 484 (2001).

[23] H. Qin, R. C. Davidson, W.W.-L. Lee, and R. Kolesnikov, Nucl. Instrum. Methods Phys. Res., Sect. A 464, 477 (2001).

[24] I. Haber, A. Friedman, D. P. Grote, S. M. Lund, S. Bernal, and R. A. Kishek, Nucl. Instrum. Methods Phys. Res., Sect. A 464, 343 (2001).

[25] R. C. Davidson, H. Qin, W.W.-L. Lee, and S. Strasburg, Nucl. Instrum. Methods Phys. Res., Sect. A 464, 358 (2001).

[26] R. C. Davidson, H. Qin, S. I. Tzenov, and E. A. Startsev, Phys. Rev. ST Accel. Beams 5, 084402 (2002).

[27] R. C. Davidson, W.W.-L. Lee, H. Qin, and E. Startsev, Phys. Plasmas 9, 340 (2002).

[28] E. A. Startsev, R. C. Davidson, and H. Qin, Phys. Plasmas 9, 3138 (2002).

[29] E. A. Startsev, R. C. Davidson, and H. Qin, Phys. Rev. ST Accel. Beams 6, 084401 (2003).

[30] L. Smith, Lawrence Berkeley Laboratory Heavy Ion Fusion Project Technical Note No. HIFAN-13, 1977.

[31] I. Hofmann, L. J. Laslett, L. Smith, and I. Haber, Part. Accel. 13, 145 (1983). 
[32] I. Hofmann, Adv. Electron. Electron Phys. Suppl. 13C, 49 (1983).

[33] J. Struckmeier, J. Klabunde, and M. Reiser, Part. Accel. 15, 47 (1984).

[34] W.W. Lee, Q. Qian, and R. C. Davidson, Phys. Lett. A 230, 347 (1997).

[35] Q. Qian, W.W. Lee, and R. C. Davidson, Phys. Plasmas 4, 1915 (1997).

[36] R. L. Gluckstern, W.-H. Cheng, and H. Ye, Phys. Rev. Lett. 75, 2835 (1995).

[37] R. L. Gluckstern, W.-H. Cheng, S. S. Kurennoy, and H. Ye, Phys. Rev. E 54, 6788 (1996).

[38] N. A. Krall and A.W. Trivelpiece, Principles of Plasma Physics (McGraw-Hill Book Co., New York, 1973).

[39] W. C. Hahn, Gen. Elec. Rev. 42, 258 (1939).
[40] S. Ramo, Phys. Rev. 56, 276 (1939).

[41] For example, see Sec. 4.8.2 of Ref. [38] and Sec. 6.2.3 of Ref. [4].

[42] I. M. Gel'fand, R. A. Minlos, and Z. Ya. Shapiro, Representations of the Rotational and Lorentz Groups and Their Applications (Pergamon Press, New York, 1963), Pt. I, Chap. 2.

[43] See Chap. 22 of Handbook of Mathematical Functions, by M. Abramowitz and I. A. Stegun, Natl. Bur. Stand. (U.S.) Circular No. 55 (U.S. GPO, Washington, DC, 1964).

[44] I. S. Gradshteyn and I. M. Ryzhik, Table of Integrals, Series, and Products (Academic Press, New York, 1965), 4th ed.

[45] See item 7.253 on p. 826 of Ref. [44]. 